\title{
Recognition of split-graphic sequences
}

\author{
Bilal A. CHAT \\ University of Kashmir \\ Hazratbal Srinagar-190006, India \\ email: bilalchat99@gmail.com \\ Shariefudddin PIRZADA \\ University of Kashmir \\ Hazratbal Srinagar-190006, India \\ email: \\ pirzadasd@kashmiruniversity.ac.in \\ Antal IVÁNYI \\ Eötvös Loránd University \\ Faculty of Informatics, H-1011 \\ Budapest, Pázmány s. 1/A, Hungary \\ email: tony@inf.elte.hu
}

\begin{abstract}
Using different definitions of split graphs we propose quick algorithms for the recognition and extremal reconstruction of split sequences among integer, regular, and graphic sequences.
\end{abstract}

\section{Basic definitions}

In this paper $a, b, l, m, n, p$ and $q$ denote nonnegative integers with $b \geq a$ and $l+m \geq 1$. We follow the terminology of Handbook of Graph Theory [28] written by Gross, Yellen and Zhang.

An $(a, b, n)$-graph is a loopless graph in which different vertices are connected at least by $\mathrm{a}$ and at most by $\mathrm{b}$ edges [43, 44]. A ( $\mathrm{b}, \mathrm{b}, \mathrm{l})$-graph is denoted by $\mathrm{K}_{\mathrm{l}}^{\mathrm{b}}$ and is called a b-clique or b-complete graph. Clearly, $\mathrm{K}_{\mathrm{l}}^{1}=\mathrm{K}_{\mathrm{l}}$, where $\mathrm{K}_{\mathrm{l}}$ is the complete graph on $\mathrm{l}$ vertices. Its complement, $\overline{\mathrm{K}}_{\mathrm{l}}$ is called independent graph on $l$ vertices.

Computing Classification System 1998: G.2.2

Mathematics Subject Classification 2010: 05C30, 05C50

Key words and phrases: psplit graph, jsplit graph, bsplit graph, graphic sequence, linear time algorithm, $(a, b, n)$-graph, potentially split sequence 
The join $[12,28,66]$ of two graphs $G$ and $H$ is denoted by $G+H$. It has the following vertex set and edge set:

$$
\mathrm{V}(\mathrm{G}+\mathrm{H})=\mathrm{V}(\mathrm{G}) \cup \mathrm{V}(\mathrm{H})
$$

and

$$
\mathrm{E}(\mathrm{G}+\mathrm{H})=\mathrm{E}(\mathrm{G}) \cup \mathrm{E}(\mathrm{H}) \cup\{\mathrm{u} v \mid u \in \mathrm{V}(\mathrm{G}) \text { and } v \in \mathrm{V}(\mathrm{H})\}
$$

A nonincreasing integer sequence $\sigma=\left(s_{1}, \ldots, s_{n}\right)$ with $s_{1} \leq b(n-1)$ and $s_{n} \geq a(n-1)$ is said $(a, b, n)$-regular $[43,44]$. A $(0, b, n)$-regular sequence shortly is said b-regular [17]. An integer sequence $\sigma$ is said $(a, b, n)$-graphic, if it is the degree sequence of an $(a, b, n)$-graph $G$ [43, 44], and such a graph $\mathrm{G}$ is referred to as a realization of $\sigma$. An integer sequence is called even, if the sum of its elements is even.

In this paper we denote the integer sequences by $\sigma$ and the degree sequences by $\delta$.

In 1965 Fulkerson, Hoffman and McAndrew [24] proposed the following definition of $(\gamma, \delta)$-multigraphs with capacity bounds. Let $n \geq 1, \delta=$ $\left(d_{1}, \ldots, d_{n}\right)$ and $\gamma=\left(c_{11}, \ldots, c_{1 n}, c_{21} \ldots, c_{2 n}, \ldots, c_{n-1, n}, c_{n, n}\right)$ sequences of nonnegative integers with $c_{i i}=0$ and $c_{i j}=c_{j i}$ for $1 \leq i<j \leq n$. Fulkerson and his coathors call $\delta$ degree vector, while $\gamma$ is the capacity vector. Let $G_{\gamma}$ denote the graph in which there is an edge between the vertex with degree $d_{i}$ and vertex with degree $d_{j}$, if $c_{i j}=1$. The capacity vector $\gamma$ has the $\boldsymbol{o d d}$-cycle condition if the graph $\mathrm{G}_{\gamma}$ has the property that any two of its odd length (simple) cycles either have a common vertices or there exists a pair of vertices, one vertex from each cycle, which are connected with an edge.

With other words, the distance between two odd length cycles is at most 1 . In particular, if $\mathrm{G}_{\gamma}$ is bipartite (has no odd length cycle) or $\mathrm{G}_{\gamma}$ is complete (all $c_{i j}$ equals to 1 ) then $\gamma$ obviously satisfies the odd-cycle condition.

An $(a, b, n)$-regular sequence is said potentially $\mathrm{K}_{\mathrm{l}}^{\mathrm{b}}$-graphic, if it has a realization $G$ containing $K_{l}^{b}$ as a subgraph. If $b=1$, then we write simply $K_{l}$ instead of $\mathrm{K}_{\mathrm{l}}^{1}$.

An $(a, b, n)$-regular sequence $\sigma=\left(s_{1}, \ldots, s_{n}\right)$ is said potentially $A_{1}^{b}$-graphic, if it has a realization $\mathrm{G}$ containing $\mathrm{J}_{l}^{\mathrm{b}}$ (definition see later) on vertices having degrees $s_{1}, \ldots, s_{l+m}$.

An $(a, b, n)$-regular sequence $\sigma=\left(s_{1}, \ldots, s_{n}\right)$ is said potentially $A_{l, m}^{b}$ graphic, if it has a realization $\mathrm{G}$ containing $\mathrm{J}_{l, m}^{\mathrm{b}}$ (definition see later) on vertices having degrees $s_{1}, \ldots, s_{l+m}$.

A $(0, b, n)$-regular sequence $\sigma=\left(s_{1}, \ldots, s_{n}\right)$ is said potentially $\mathrm{J}_{l, m}^{\mathrm{b}}$-graphic if it has a realization $\mathrm{G}$ containing $\mathrm{J}_{l, m}^{\mathrm{b}}$ (definition see later) on vertices having 
degrees $s_{1}, \ldots, s_{l+m}$. If $b=1$, then we write simply $A_{l}$ insted $A_{b}^{1}, A_{l, m}^{1}$ instead of $A_{l, m}^{b}, J_{l, m}$ instead of $J_{l, m}^{1}$, and $J_{l, m}$ instead of $J_{l, m}^{1}$.

Let $n \geq 2$ and $\sigma=\left(s_{1}, \ldots, s_{n}\right)$ be a nonnegative integer sequence, and $k$ be any integer $1 \leq \mathrm{k} \leq \mathrm{n}$. Let $\sigma^{\prime}=\left(\mathrm{s}_{1}^{\prime}, \ldots, \mathrm{s}_{\mathrm{n}}^{\prime}\right)$ be the sequence obtained from $\mathrm{s}$ by setting $s_{k}=0$ and $s_{i}^{\prime}=s_{i}-1$ for the $s_{k}$ largest elements of $s$ other than $s_{k}$. Let $H_{k}$ be the graph obtained on the vertex set $V=\left\{v_{1}, \ldots, v_{n}\right\}$ by joining $v_{k}$ to the $s_{k}$ vertices corresponding to the $s_{k}$ elements used to obtain $s^{\prime}$. This operation of getting $s^{\prime}$ and $H_{k}$ is called laying off $s_{k}, s^{\prime}$ is called residual sequence, and $H_{k}$ is called the subgraph obtained by laying off $s_{k}$ [51].

Now we formulate several definitions of split graphs.

The classical and most distributed definition of split graphs was introduced by Földes and Hammer in 1977 [21, 22, 26, 28].

Definition 1 (Földes, Hammer [21, 22]) An (l, m)-partitioned split graph (shortly: psplit graph) is one whose vertex set can be partitioned into two disjoint subsets spanning a clique $\mathrm{K}_{\mathrm{l}}$ and an independent graph $\overline{\mathrm{K}}_{\mathrm{m}}$. It is denoted by $\mathrm{S}_{\mathrm{l}, \mathrm{m}}$.

It is worth to mention that one of $l$ and $m$ can be zero, that is if $l \geq 1$, then $S_{l, 0}$ is also a psplit graph, and if $m \geq 1$, then $S_{0, m}$ is also a psplit graph, and the independent graph $\overline{\mathrm{K}}_{0, \mathrm{~m}}=\mathrm{S}_{0, \mathrm{~m}}$ are also psplit graphs. For the number of edges $\left|E\left(S_{l, m}\right)\right|$ of an $S_{l, m}$ hold the inequalities $l(l-1) / 2 \leq\left|E\left(S_{l, m}\right)\right| \leq l(l-1) / 2+l \cdot m$ and between these bounds every integer value is realizable.

Consider the following example (Figure 1). Let $G=(\mathrm{V}, \mathrm{E})$, where $\mathrm{V}=$ $\left\{v_{1}, \ldots, v_{5}\right\}$ and $E=\left(v_{1} v_{2}, v_{1} v_{3}, v_{1} v_{4}, v_{2} v_{3}, v_{2} v_{4}, v_{3} v_{4}\right\}$, that is $G$ contains six edges. Then $G$ is $S_{3,2}$ and also $S_{4,1}$ due to the following two partitions of $V$ : $\left\{v_{1}, v_{2}, v_{3}, v_{4}\right\}$ plus $\left\{v_{4}, v_{5}\right\}$ (Figure 1a)) containing all six edges and also is $S_{3,2}$ due to the partition $\left\{v_{1}, v_{2}, v_{3}\right\}$ plus $\left\{v_{4}, v_{5}\right\}$ (Figure 1b)) containing only three edges.

In 1996 Brandstädt introduced the following definition of $(l, m)$-multipartitioned split graphs. Let $G=(V, E)$ with $|V|=n . V_{1}, \ldots, V_{k}$ is a partition of $V$, if and only if for all $u, v \in\{1, \ldots, k\}$ with $i<j V_{i} \cap V_{j}=\emptyset$ and $\bigcup_{i=1}^{k} V_{i}=V$. A partition $C_{1}, \ldots, C_{m}, I_{1}, \ldots, I_{m}$, with cliques $C_{i}, i \in\{1, \ldots, l\}$ and independent sets $I_{j}, j \in\{1, \ldots, m\}$ is an $(l, m)$-partition of $V$.

Definition 2 (Brandstädt $[7,8,10])$ A graph $\mathrm{G}=(\mathrm{V}, \mathrm{E})$ is called an $(\mathrm{l}, \mathrm{m})$ split graph, if its vertex set has an $(\mathrm{l}, \mathrm{m})$-partition.

In 1998 Gyárfás generalized (l, m)-psplit graphs to (l, m)-bsplit graphs. 


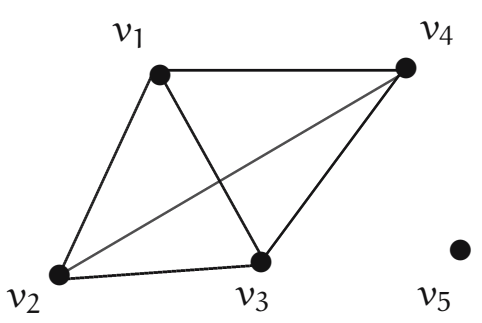

(a)

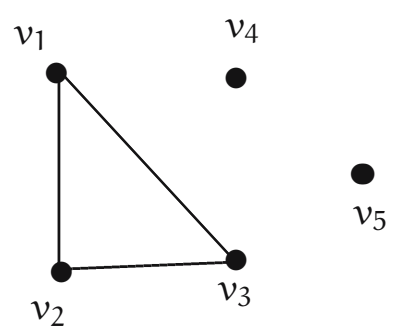

(b)

Figure 1: Partition of a psplit graph is not unique.

Definition 3 (Gyárfás [30]) A graph $\mathrm{G}$ is called (l, m)-bounded split graph (shortly: bsplit graph) if its vertex set can be partitioned into $\mathrm{A}$ and $\mathrm{B}$ so that the order of the largest clique graph in $\mathrm{A}$ is $\mathrm{l}$ and the order of the largest complete subgraph in $\mathrm{B}$ is $\mathrm{m}$.

In 2001 Hell, Klein, Protti, and Tito [41] defined (k, l)-graphs so, that their vertex set can be partitioned into $k$ cliques and $l$ independent sets.

In 2005 Bradstädt, Hammer, Le and Lozin studied bisplit graphs, defined as follows.

Definition 4 (Brandstädt, Hammer, Le, Lozin [9]) A graph $\mathrm{G}$ is called (l, m)bisplit graph (shortly: bisplit graph) if its vertex set can be partitioned into a complete bipartite graph and an independent graph. It is denoted by $\mathrm{B}_{l, m}$.

For the number $\left|E\left(B_{l, m}\right)\right|$ of edges of a bisplit graph $B_{l, m}$ hold the inequalities $l^{2} \leq\left|E\left(B_{l, m}\right)\right| \leq l^{2}+2 l m$.

In 2007 Le and Ritter [55] defined probe split graphs (modifying the definition of interval split graphs [61]).

Definition 5 (Le, Ritter [55]) A G(V,E) graph is probe split graphs, if $\mathrm{V}$ can be partitoned into two parts $\mathrm{N}$ (nonprobes) and $\mathrm{P}$ (probes) where $\mathrm{N}$ is an independent set and there exists $\mathrm{E}^{\prime} \subset \mathrm{N} \times \mathrm{N}$ such that $\mathrm{G}^{\prime}=\left(\mathrm{V}, \mathrm{E} \cup \mathrm{E}^{\prime}\right)$ is a psplit graph.

In 2009 [6] Boros, Gurvich and Zverovich [6] proposed the definition of almost CIS-graphs.

The following simple definition appeared in 2011 and later in the papers of different authors as Chat, Pirzada, and Yin [67, 86, 87]. 
Definition 6 (Yin $[86,87])$ An (l, m)-join split graph (shortly: jsplit graph) is the join of $\mathrm{K}_{\mathrm{l}}$ and $\overline{\mathrm{K}}_{\mathrm{m}}$. It is denoted by $\mathrm{J}_{\mathrm{l}, \mathrm{m}}$.

It is worth to remark, that jsplit graphs are special cases of psplit graphs: if $\mathrm{G}$ is a jsplit graph, then any vertex of $\mathrm{K}_{\mathrm{l}}$ is connected with any vertex of $\overline{\mathrm{K}}_{\mathrm{m}}$, while in the corresponding psplit graph even all such edges can be absent.

Consider the following example Let $\mathrm{H}=(\mathrm{V}, \mathrm{E})$, where $\mathrm{V}=\left\{v_{1}, \ldots, v_{5}\right\}$ and $\mathrm{E}=\left(v_{1} v_{2}, v_{1} v_{3}, v_{1} v_{4}, v_{1} v_{5}, v_{2} v_{3}, v_{2} v_{4}, v_{2} v_{5}, v_{3} v_{4}, v_{3} v_{5}, v_{4} v_{5}\right\}$, that is $\mathrm{H}$ is a clique on 5 vertices and so it contains ten edges. Then $\mathrm{H}$ is $\mathrm{J}_{5,0}$ and also $\mathrm{J}_{4,1}$ due to the following partition of $\mathrm{V}:\left\{v_{1}, v_{2}, v_{3} . v_{4}, v_{5}\right\}$ plus $\emptyset$ and is $\mathrm{J}_{4,1}$ for example due to the partition $\left\{v_{1}, v_{2}, v_{3}, v_{4}\right\}$ plus $\left\{v_{5}\right\}$. We can remark that these partitions at the same time give psplit graphs with the same size parameters.

Let $\mathrm{K}_{\mathrm{l}}^{\mathrm{b}}$ and $\mathrm{K}_{\mathrm{m}}^{\mathrm{b}}$ be $\mathrm{b}$-cliques, and let $\overline{\mathrm{K}}_{\mathrm{m}}^{\mathrm{b}}$ be the complement of $\mathrm{K}_{\mathrm{m}}^{\mathrm{b}}$, that is an empty graph on $m$ vertices. We propose the following generalization of psplit-graphs.

Definition $7 A$ (b,l,m)-partitioned split graph (shortly: b-psplit graph) is one whose vertex set can be partitioned into two disjoint subsets spanning a $\mathrm{b}$-clique $\mathrm{K}_{\mathrm{l}}^{\mathrm{b}}$ and an empty graph $\overline{\mathrm{K}}_{\mathrm{m}}^{\mathrm{b}}$. It is denoted by $\mathrm{S}_{\mathrm{l}, \mathrm{m}}^{\mathrm{b}}$.

Clearly, $S_{l, m}^{1}=S_{l, m}$.

In 2011 Yin extended the definition of the jsplit-graphs to b-jsplit graphs.

Definition 8 (Yin [87]) A (b, l, m)-join-split graph (shortly: b-jsplit graph) is the join of $\mathrm{K}_{\mathrm{l}}^{\mathrm{b}}$ and $\overline{\mathrm{K}}_{\mathrm{m}}^{\mathrm{b}}$. It is denoted by $\mathrm{J}_{\mathrm{l}, \mathrm{m}}^{\mathrm{b}}$.

Clearly, $\mathrm{J}_{\mathrm{l}, \mathrm{m}}^{1}=\mathrm{J}_{\mathrm{l}, \mathrm{m}}$.

Figure 2 shows $\mathrm{J}_{3,2}$ (part a) and $\mathrm{J}_{3,2}^{2}$ (part b).

The structure of the paper is as follows. After the basic definition (Section 1) in Section 2, 3 and 4 the most important mathematical background results connected with graphical, potentially graphical and potentially split graphical sequences are reviewed, then in Sections 5, 6 the new mathematical results are presented.

We review the known algorithms in Sections 7 and 8, while the now proposed algorithms are presented in Section 9.

The main results of the paper are that using different definitions of split graphs $[4,5,7,8,9,10,21,22,23,26,28,30,67,82,86,87,88]$ we propose quick algorithms for the recognition and extremal reconstruction of split sequences among integer, regular $[17,45]$ and graphic $[43,45,48]$ sequences. 


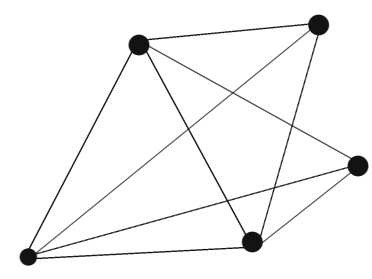

a)

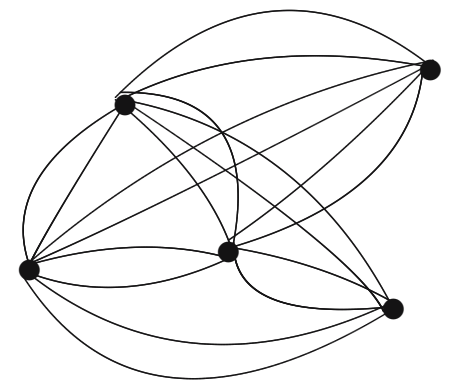

b)

Figure 2: Jsplit graphs $\left.\mathrm{J}_{3,2}(\mathrm{a})\right)$ and $\left.\mathrm{J}_{3,2}^{2}(\mathrm{~b})\right)$.

\section{Known results on graphic sequences}

In 1955 Havel, in 1962 Hakimi proposed the following necessary and sufficient condition for $n$-regular sequences to be graphic.

Theorem 9 (Havel [32], Havel [36]) Let $\mathrm{n} \geq 2$. An $\mathrm{n}$-regular sequence $\sigma=$ $\left(s_{1}, \ldots, s_{n}\right)$ is graphical if and only if the sequence $\sigma^{\prime}=\left(s_{2}-1, s_{3}-1, \ldots, s_{s_{1}}-\right.$ $\left.1, s_{s_{1}+1}-1, s_{s_{1}+2}, \ldots, s_{n-1}, s_{n}\right)$ is graphical.

Proof. See Hakimi[32], Havel [36].

The recursive algorithm implementing this theorem requires in worst case $\Theta\left(n^{2}\right)$ time. It is worth to remark, that this algorithm not only tests the sequences, but if they are graphic, the algorithm constructs a realization of the tested sequence.

In 1973 Kleitman and Wang improved the Havel-Hakimi theorem: according to their following theorem it is sufficient to test any nonzero element of the input sequence. The central element of their proof is the laying off the tested sequence.

Theorem 10 (Kleitman, Wang [51]) Let $\mathrm{n} \geq 2$. A nonnegative integer sequence $\sigma$ is graphic if and only if the residual sequence obtained by laying off any nonzero element of $\mathrm{s}$ is graphic.

Proof. See Kleitman [51].

In 1974 Chungphaisan [13] extended the definition of laying off and residual sequence to b-laying off and b-residual sequence as follows. Let $\sigma=$ 
$\left(s_{1}, \ldots, s_{n}\right)$ be an $n$-regular sequence and $1 \leq k \leq n$. Define $\sigma_{k}^{\prime}=\left(s_{1}^{\prime}, \ldots, s_{n-1}^{\prime}\right)$ to be the nonincreasing rearrangement of the sequence obtained from $\left(s_{1}, \ldots\right.$, $\left.s_{k-1}, s_{k+1}, \ldots, s_{n}\right)$ reducing by 1 the remaining largest term that has not already been reduced $b$ times, and repeating the procedure $s_{k}$ times. $s_{k}^{\prime}$ is called the b-residual sequence obtained from $\sigma$ by b-laying off $s_{k}$.

Using the b-laying off operation Chungphaisan proved the following generalization of Kleitman-Wang theorem.

Theorem 11 (Chungphaisan [13]) Let $\mathrm{n} \geq 2$. A nonnegative integer sequence $\sigma$ is $\mathrm{b}$-graphic if and only if the $\mathrm{b}$-residual sequence obtained by b-laying off any nonzero element of $\sigma$ is graphic.

Proof. See Chungphaisan [13].

In 1960 Erdős and Gallai gave the following necessary and sufficient condition.

Theorem 12 (Erdős, Gallai [17]) Let $\mathrm{n} \geq 1$. An $\mathrm{n}$-regular even sequence $\sigma=\left(s_{1}, \ldots, s_{n}\right)$ is graphical if and only if

$$
\sum_{i=1}^{k} d_{i} \leq k(k-1)+\sum_{i=k+1}^{n} \min \left(d_{i}, k\right)
$$

is satisfied for each integer $\mathrm{k}, 1 \leq \mathrm{k} \leq \mathrm{n}$.

Proof. See Erdős, Gallai [17].

Later several new proofs of this theorem were published, among others by to Gasharov in 1997, [25], by Tripathi and Tiagy in 2008 [77], by Tripathi, Venugopalan and West in 2010 [78].

In 1974 Chungphaisan extended Erdős-Gallai theorems to (0, b, n)-graphs.

Theorem 13 (Chungphaisan [13]). Let $\sigma=\left(\mathrm{s}_{1}, \ldots, \mathrm{s}_{\mathrm{n}}\right)$ be an $(\mathrm{a}, \mathrm{b}, \mathrm{n})$-regular even sequence. Then $\sigma$ is $(0, b, n)$-graphic if and only if for each positive integer $\mathrm{t} \leq \mathrm{n}$,

$$
\sum_{i=1}^{t} s_{i} \leq b t(t-1)+\sum_{i=t+1}^{n} \min \left(b t, s_{i}\right) .
$$

Proof. See Chungphaisan [13].

We remark then if we use the theorems of Erdös-Gallai [17], Havel-Hakimi [32, 36], Kleitman-Wang [51] or Chungphaisan [13] to decide whether an integer sequence is graphic, the decision requires quadratic time. In 2012 Iványi 
proposed an algorithm for $(0, b, n)$ graphs, then in 2012 [45] for ( $a, b, n)$-graphs allowing the decision in worst case in $\mathrm{O}(n)$ time.

In the worst case the algorithm based on Theorem 13 requires quadratic time, but the following assertion allows us to test the sequences in linear time. Since this is an important result, we repeat its proof.

Theorem 14 (Iványi [45]) If $\mathrm{n} \geq 1$, then the $\sigma=\left(\mathrm{s}_{1}, \ldots, \mathrm{s}_{\mathrm{n}}\right)(0, \mathrm{~b}, \mathrm{n})$-regular sequence is $(0, \mathrm{~b}, \mathrm{n})$-graphic if and only if

$$
\sum_{i=1}^{n} s_{i} \text { is even }
$$

and

$$
H_{i}>b i\left(y_{i}-1\right)+H_{n}-H_{y} \quad(i=1, \ldots, n-1),
$$

where

$$
y_{i}=\max \left(i, w_{i}\right) \quad(i=1, \ldots, n-1) .
$$

Proof. This proof is an improved version of the proof of linearity of EGL in [48] and was published in 2012 [45]

We exploit that $s$ is monotone and determine the capacity estimations $c_{k}=\min \left(j b, s_{k}\right)$ appearing in (1) in constant time. The base of the quick computation is again the sequence of the weight points $w(\sigma)=\left(w_{1}, \ldots, w_{n-1}\right)$ containing the weight points belonging to the elements of $\sigma$, and the sequence $y(\sigma)=\left(y_{1}, \ldots, y_{n}\right)$ containing the cutting points of the elements of s. For given $s_{i}$ the weight point $w_{i}$ is the largest $k(1 \leq k \leq n)$ having the property $s_{k} \geq i$. The cutting point $y_{i}$ belonging to $s_{i}$ is the maximum of $i$ and $w_{i}$, see (2).

During the testing of the elements of $s$ there are two cases:

a) if $i>w_{i}$, then the maximal contribution $C_{i}=\sum_{k=i+1}^{n} \min \left(i, s_{k}\right)$ of the actual tail of $s$ is at most $H_{n}-H_{i}$, since the maximal contribution $c_{k}=$ $\min \left(i, s_{k}\right)$ of the element $s_{k}$ is only $s_{k}$, and so

$$
\mathrm{C}_{\mathrm{i}}=\sum_{\mathrm{k}=\mathrm{i}+1}^{\mathrm{n}} \mathrm{c}_{\mathrm{k}}=\mathrm{H}_{\mathrm{n}}-\mathrm{H}_{\mathrm{i}} \text {, }
$$

implying the requirement

$$
\mathrm{H}_{\mathrm{i}} \leq \mathrm{bi}(\mathrm{i}-1)+\mathrm{H}_{\mathrm{n}}-\mathrm{H}_{i}
$$


b) if $i \leq w_{i}$, then the maximal contribution $C_{i}$ of the actual tail of $s$ consists of contributions of two types: $c_{i+1}, \ldots, c_{w_{i}}$ are equal to bi, while $c_{j}=s_{j}$ for $\mathrm{j}=w_{\mathrm{i}}+1, \ldots, \mathrm{n}$, therefore we have

$$
C_{i}=b i\left(w_{i}-i\right)+H_{n}-H_{w_{i}}
$$

implying the requirement

$$
H_{i}=b i(i-1)+b i\left(w_{i}-i\right)+H_{n}-H_{w_{i}} .
$$

Transforming (5) we get

$$
\mathrm{H}_{\mathrm{i}}=\operatorname{bi}\left(w_{\mathrm{i}}-1\right)+\mathrm{H}_{\mathrm{n}}-\mathrm{H}_{w_{i}} .
$$

Considering the definition of $y_{i}$ given in (2), further (4) and (5) we get the required (1).

In 1981 Rao [69] analyzed the conditions for graphic sequences to be Pgraphic.

In 2009 Hell Hell and Kirkpatrick [40] extended the concept of graphic sequences defining quasi-graphic sequences and proposing a linear time algorithm for their certifying. A state of art of certifying algorithms was published in 2011 [59] by McConell, Mehlhorn, Näher and Schweitzer.

The following assertion is the base of the quick testing of integer sequences whether they are $(a, b, n)$-graphic or not. In 2012 Iványi proved that theorem of Chungphaisan has the following consequence allowing the quick test of potential $(a, b, n)$-graphic sequences..

Corollary 15 (Iványi [45]) If $\mathrm{n} \geq 2$, then an $(\mathrm{a}, \mathrm{b}, \mathrm{n})$-regular sequence $\sigma=$ $\left(\mathrm{s}_{1}, \ldots, \mathrm{s}_{\mathrm{n}}\right)$ is $(\mathrm{a}, \mathrm{b}, \mathrm{n})$-graphic if and only if the sequence $\mathrm{s}^{\prime}=\left(\mathrm{s}_{1}-\mathrm{a}, \mathrm{s}_{2}-\right.$ $\left.\mathrm{a}, \ldots, \mathrm{s}_{\mathrm{n}}-\mathrm{a}\right)$ is $(0, \mathrm{~b}-\mathrm{a}, \mathrm{n})$-regular.

Proof. See [45]

This corollary allows the testing of $(a, b, n)$-regular sequences in worst case in $\mathrm{O}(\mathrm{n})$ time using algorithm ERDős-GALLAI-LINEAR [48] or algorithm HAVELHakimi-Chungphaisan [45].

The following sources contain results on the enumeration of graphic and b-graphic sequences [35, 45, 46, 47, 48, 49, 74].

\section{Known results on A-graphic sequences}

The following two results due to J. H. Yin are generalizations from 1-graphs to b-graphs of two well-known results given by A. R. Rao $[50,68,70]$ 
Theorem 16 (Yin [84]). Let $\mathrm{n} \geq \mathrm{l}+1$ and $\sigma=\left(\mathrm{s}_{1}, \ldots, \mathrm{s}_{\mathrm{n}}\right)$ be a b-graphic sequence with $s_{l+1} \geq \mathrm{bl}$. Then $\sigma$ is potentially $A_{l+1}^{b}$-graphic if and only if $s_{l+1}^{\prime}$ is b-graphic.

Proof. See [84].

Theorem 17 (Yin [84]) Let $\mathrm{n} \geq \mathrm{l}+1$ and $\sigma=\left(\mathrm{s}_{1}, \ldots, \mathrm{s}_{\mathrm{n}}\right)$ be a b-graphic sequence with $s_{l+1} \geq 2 \mathrm{bl}-1$, then $\mathrm{s}$ is potentially $\mathrm{A}_{\mathrm{l}+1}^{\mathrm{b}}$-graphic.

Proof. See [84].

In 1978 Hakimi and Schmeichel [33] studied potentially and forcibly Pgraphic sequences.

In 2009 Yin generalized a result Gould, Jacobson and Lehel [27].

Theorem 18 (Yin [85]) If $\delta=\left(\mathrm{d}_{1}, \ldots, \mathrm{d}_{\mathrm{n}}\right)$ is is a b-graphic sequence with a realization $\mathrm{G}$ containing a b-graph $\mathrm{H}$ as a subgraph, then there exists a realization $\mathrm{G}^{\prime}$ of $\delta$ so that the vertices of $\mathrm{H}$ have the largest degrees of $\delta$.

Proof. See [85].

In 2009 Yin wrote [84] that the following assertion is a special case of Theorem 18.

Corollary 19 A b-graphic sequence is potentially $\mathrm{K}_{\mathrm{l}}^{\mathrm{b}}$-graphic, if and only if it is potentially $A_{\mathrm{l}}^{\mathrm{b}}$-graphic.

Proof. We prove a bit stronger assertion.

It is trivial, that if an integer sequence is $A_{l}^{b}$-graphic, then it is b-graphic.

The sufficiency can be proved following the proof of Lemma 2.1. in [85].

We remark, that Theorem 18 contains only a sufficient condition.

Let $l, m, r$ and $n$ be positive integers, $n \geq l+m$, and let $\sigma=\left(s_{1}, \ldots, s_{n}\right)$ be an $n$-regular sequence with $s_{l} \geq l+m-1$ and $s_{l+m} \geq l$. We construct the sequences $\sigma_{1}, \ldots, \sigma_{l}$ as follows. At first we construct the sequence

$$
\sigma_{1}=\left(s_{1}-1, \ldots, s_{l}-1, s_{m+1}, s_{l+1}, \ldots, s_{l+m+1}^{1}, \ldots, s_{n}^{1}\right)
$$

from $\sigma$ by deleting $s_{1}$, reducing the first $s_{1}$ remaining elements of $\sigma$ by one, and then reordering the last $n-l-m$ elements to be nonincreasing. For $2 \leq i \leq l$, we recursive construct

$$
\sigma_{i}=\left(s_{i+1}-i, \ldots, s_{l}-i, s_{l+1}-i, \ldots, s_{l+1}-i, s_{l+m+1}^{i-1}, \ldots, s_{n}^{i}\right)
$$


from $\sigma_{i-1}$ by deleting $s_{i}-i+1$, reducing the first $s_{i}-i+1$ remaining elements of $\sigma_{i-1}$ by one, and then reordering the last $n-l-m$ elements to be nonincreasingIn 2012 Yin proved the two following theorems.

In 2012 Yin proved the following two theorems.

Theorem 20 (Yin [87]) $\sigma$ is potentially $A_{b}$-graphic if and only if $\sigma_{b}$ is graphic.

Proof. See [87].

Theorem 21 (Yin [87]) Let $\mathrm{n} \geq \mathrm{l}+\mathrm{m}$ and let $\sigma=\left(\mathrm{s}_{1}, \ldots, \mathrm{s}_{\mathrm{n}}\right)$ be a nonincreasing graphic sequence. If $\mathrm{s}_{\mathrm{l}+\mathrm{m}} \geq \mathrm{l}+\mathrm{m}-2$, then $\sigma$ is potentially $\mathrm{A}_{\mathrm{l,m}-\mathrm{m}}$ graphic.

Proof. See [87].

Using the algorithm ERDös-GALLAI-LinEar [48] or algorithm HAVEL-HAKIMI-LiNEAR [45] we can decide in worst case in $\mathrm{O}(\mathrm{n})$ time whether $\pi$ is graphic.

The following theorem allows to decrease the expected running time of HAVEL-HAKIMI-SPLIT.

Theorem 22 (Yin [87]). Let $\mathrm{n} \geq \mathrm{l}+\mathrm{m}$ and let $\sigma=\left(\mathrm{s}_{1}, \ldots, \mathrm{s}_{\mathrm{n}}\right)$ be an $\mathrm{n}$ -

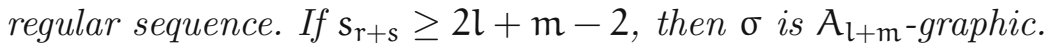

Proof. See [87].

In the same paper Yin [87] published a Havel-Hakimi type algorithm which constructs the corresponding $\mathrm{J}_{\mathrm{l}, \mathrm{m} \text {-graph. }}$

Let $A_{n}=\left(a_{1}, \ldots, a_{n}\right)$ be an $n$-regular sequence, and $B_{n}=\left(b_{1}, \ldots, b_{n}\right)$ a sequence of nonnegative integers with $a_{i} \leq b_{i}$ and $a_{i}+b_{i} \geq a_{i+1}+b_{i+1}$ for $i=1, \ldots, n-1$. $\left(A_{n} ; B_{n}\right)$ is said to be potentially $K_{m+1}$-graphic (resp.

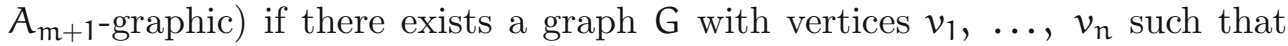
$a_{i} \leq v_{i}(G) \leq b_{i}$ for $i=1, \ldots, n$ and $G$ contains $K_{m+1}$ as a subgraph. In 2013 Yin [88] characterized $\left(A_{n} ; B_{n}\right)$ so, that it is potentially $A_{m+1}$-graphic and potentially $\mathrm{K}_{\mathrm{m}+1 \text {-graphic. }}$

In 2014 Yin [89] characterized the sequences having a realization containing an arbitrary subgraph.

In 2014 Pirzada and Chat proved the following assertion.

Theorem 23 (Pirzada, Chat [67]) If $\mathrm{G}_{1}$ is a realization of $\sigma_{1}=\left(s_{1}^{1}, \ldots, s_{l}^{1}\right)$, containing $\mathrm{K}_{\mathrm{l}}$ as a subgraph and $\mathrm{G}_{2}$ is a realization of $\sigma_{2}=\left(\mathrm{s}_{1}^{2}, \ldots, \mathrm{s}_{\mathrm{m}}^{2}\right)$ containing $\mathrm{K}_{\mathrm{m}}$ as a subgraph, then the degree sequence $\sigma=\left(s_{1}, \ldots, s_{l+\mathrm{m}}\right)$ of the

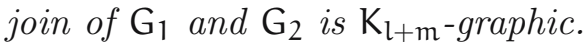


Proof. See [67]

\section{Known results on split sequences}

The girth $\mathrm{g}(\mathrm{G})$ of a graph $\mathrm{G}$ containing at last one cycle is the length of its shortest cycle. The girth of an acyclic graph is infinite. A graph $G$ is called chordal, if it does not contain an induced subgraph with finite girth greater then 3.

In 1977 Földes and Hammer gave the following characterization of psplit graphs.

Theorem 24 (Földes, Hammer [22]) For any graph G the following three conditions are equivalent:

(i) $\mathrm{G}$ and $\overline{\mathrm{G}}$ both are chordal;

(ii) $\mathrm{V}(\mathrm{G})$ can be partitioned into a complete and an empty set;

(iii) $\mathrm{G}$ does not contain an induced subgraph isomorphic to $2 \mathrm{~K}_{2}, \mathrm{C}_{4}$ or $\mathrm{C}_{5}$.

In 1993 Blázsik, Hujter, Pluhár and Tuza [5] characterized the pseudo split graphs defined as graphs with no induced $\mathrm{C}_{4}$ and $2 \mathrm{~K}_{2}$ (see also [2]). In 1998 Maffray and Preissmann [58] proved the following assertion.

Theorem 25 (Maffray, Preissmann [58]) G is a pseudo split graph with a nonincreasing degree sequence $\delta=\left(\mathrm{d}_{1}, \ldots, \mathrm{d}_{\mathfrak{n}}\right)$, then $\mathrm{G}$ is a pseudo split graph, if $\mathrm{G}$ is a split graph or

$$
\sum_{i=1}^{q} d_{i}=q(q+4)+\sum_{i=m+1}^{n} d_{i}
$$

and

$$
\mathrm{d}_{\mathrm{q}+1}=\mathrm{d}_{\mathrm{q}+2}=\mathrm{d}_{\mathrm{q}+3}=\mathrm{d}_{\mathrm{q}+4}=\mathrm{d}_{\mathrm{q}+5}=\mathrm{q}+2,
$$

where $\mathrm{q}=\max \left(\boldsymbol{i} \mid \mathrm{d}_{\mathrm{i}} \geq \mathrm{q}+4\right)$.

Proof. See [58].

The following theorem allows to design a linear time algorithm recognising the psplit graphs in linear time.

Theorem 26 (Golumbic [26]; Hammer, Simeone [34]; Tyshkevich [79]; Tyshkevich, Melnikow, Kotov [81], Wikipedia [82]) Let the nonincreasing degree sequence of a graph $\mathrm{G}$ be $\delta=\left(\mathrm{d}_{1}, \ldots, \mathrm{d}_{\mathrm{n}}\right)$, and let $\mathrm{m}$ be the largest value of $\mathrm{i}$ 
such that $\mathrm{d}_{\mathrm{i}} \geq \mathrm{i}-1$. Then $\mathrm{G}$ is a psplit graph if and only if

$$
\sum_{i=1}^{m} d_{i}=m(m-1)+\sum_{i=m+1}^{n} d_{i} .
$$

If this is the case, then the $\mathrm{m}$ vertices with the largest degrees form a maximum clique in $\mathrm{G}$, and the remaining vertices constitute and independent set.

Proof. See [34].

An extremal problem for 1-graphic sequences to be potentially $K_{l}^{1}$-graphic was considered by Erdős, Jacobson and Lehel [19], and solved by Gould et al. [27] and Li et al. [56, 57]. Recently, Yin [85] generalized this extremal problem and the Erdős-Jacobson-Lehel conjecture from 1-graphs to b-graphs.

Different split graphs are closely connected with the problems of colorings of graphs, since the clique number gives a lower bound of coloring number. E.g. Erdős and Gyárfás [18], Gyárfás and Lehel [29] deal with coloring of psplit graphs. Yin and $\mathrm{Li}$ [90] gave sufficient conditions for graphic sequences to have a realization with prescribed clique size.

There are many publications on the maximal clique algorithms. Recently Zavalnij [91] analysed parallel algorithms for the the solution of the maximal clique problem. This problem was earlier studied e.g. in $[60,63,64,75,72,73$, 76].

In 2000 observed a bijection between nonisomorphic psplit graphs and minimal covers of a set by its subsets. Using the formula proved by Clarke [15] for the number of minimal covers, Royle [71] proved the following assertion, giving the number $p(n)$ of the nonisomorphic psplit graphs on $n$ vertices. This result was published also by Tyshkevich and Chernak in 1990 .

Theorem 27 (Royle [71], Tyshkevich, Chernak [80]) If $n \geq 1$, then

$$
p(n)=\sum_{k=1}^{n} t(n-k, k),
$$

where

$$
\begin{gathered}
t(n, k)=\frac{1}{n ! k !} \sum_{\alpha \in P_{n}, \beta \in P_{k}}\left(\begin{array}{l}
n \\
\alpha
\end{array}\right)\left(\begin{array}{l}
\beta \\
k
\end{array}\right) \prod_{i}\left(\left(\prod_{j} 2^{\left(\alpha_{i}, \beta_{j}\right)}\right)\right), \\
\left(\begin{array}{l}
n \\
\alpha
\end{array}\right)=\frac{n !}{\prod_{i} \mu_{i} ! i \mu_{i}}
\end{gathered}
$$


where $\mu_{i}$ is the number of $i$ 's in the partition $\alpha,(u, v)$ denotes the greatest common divisor of $\mathrm{u}$ and $v, \mathrm{P}_{\mathrm{n}}$ is the set of all partitions of $\mathrm{n}$.

Figure 3 contains the values of $p(n)$ for $n=1, \ldots, 20$. This data are taken from Encyclopedia of Interger Sequences [39] containing the values of $p(n)$ for $\mathrm{n}=1, \ldots, 40$ vertices.

\begin{tabular}{||r|r|r|r||}
\hline $\mathrm{n}$ & $\mathrm{p}(\mathrm{n})$ & $\mathrm{n}$ & $\mathrm{p}(\mathrm{n})$ \\
\hline 1 & 1 & 11 & 64956 \\
\hline 2 & 2 & 12 & 501696 \\
\hline 3 & 4 & 13 & 5067146 \\
\hline 4 & 9 & 14 & 67997750 \\
\hline 5 & 21 & 15 & 1224275498 \\
\hline 6 & 56 & 16 & 29733449510 \\
\hline 7 & 164 & 17 & 976520265678 \\
\hline 8 & 557 & 18 & 43425320764422 \\
\hline 9 & 2223 & 19 & 2616632636247976 \\
\hline 10 & 10766 & 20 & 213796933371366930 \\
\hline
\end{tabular}

Figure 3: The number $p(n)$ of nonisomorphic psplit graphs for $n=1, \ldots, 20$ vertices.

In 1995 Nikolopoulos proposed a constant-time parallel algorithm for the recognition of psplit graphs.

Theorem 28 (Nikolopoulos [62]) Let $\mathrm{G}(\mathrm{V}, \mathrm{E})$ be a graph with $|\mathrm{V}|=\mathrm{n}$ and $|\mathrm{E}|=\mathrm{m}$. Then algorithm SPLIT-RECOGNITION decides-using $\mathrm{O}(\mathrm{nm})$ processors - in $\mathrm{O}(1)$ time whether $\mathrm{G}$ is a psplit graph.

Proof. See [62].

Several papers deals with the hamiltonicity of split graphs. E.g. in 1980 Burkard and Hammer [11] gave a necessary condition of the hamiltonicity of psplit graphs.

In 1988 Peemüller analyzed the condition of Burkard and Hammer and proved new necessary conditions for hamiltonian psplit graphs.

Theorem 29 (Peemüller [65]) Let $\mathrm{G}=\left(\mathrm{C}, \mathrm{I}, \mathrm{E}_{1}, \mathrm{E}_{2}\right)$ be a psplit graph with $|\mathrm{C}|<|\mathrm{I}|$. If $\mathrm{G}$ is hamiltonian, then

$$
\left.2\left|X^{\prime}\right|-m\left(X^{\prime}, Y^{\prime}\right)+f\left(X^{\prime}, Y^{\prime}\right) \leq m\left(Y^{\prime}, \bar{Y}\right)-f Y^{\prime}, \bar{Y}\right),
$$

for all $\mathrm{X}^{\prime} \subset \mathrm{X}, \mathrm{X}^{\prime} \neq \emptyset$, while $\mathrm{m}, \mathrm{f}$ and $\mathrm{N}$ is defined in [65]. 
Proof. See [65].

In 1998 Woeginger proved the following property of the taughness [14] of psplit graphs solving a problem posed by Kratsch, Lehel and Müller in 1996 [53].

Theorem 30 (Woginger [83]) The toughness of psplit graphs can be computed in polynomial time.

Proof. See [83].

It is worth to remark that in 1990 Burkard, Hakimi and Schmeichel [3] that recognising of the toughness of a graph is NP-hard.

In 1999 Brandstädt, Le and Spinrad [10], in 2012 Almeida, Mello and Morgana [1] studied the classification problem of split graphs.

In 2006 Kratsch, McConnell, Mehlhorn, and Spinrad [52] reviewed certifying algorithms for recognizing interval graphs and permutation graphs

In 2008 Ibarra [42] studied fully dynamical algorithms of maintenance of psplit graphs.

In 2009 Heggernes and Mancini [38] analysed the minimal completion of psplit graphs.

In 2012 LaMar [54] defined directed psplit graphs and derived conditions for integer sequences to be degree sequences of directed psplit graphs.

In 2014 Habib and Mamcarz [31] investigated split decompositions of graphs.

\section{New results for $A$-graphic sequences}

In the next result, we use the Havel-Hakimi procedure to test whether a bgraphic sequence $\delta$ is potentially $A_{l, m}^{b}$-graphic.

Theorem 31 Let $\mathrm{b} \geq 1$ and $\mathrm{n} \geq 1$. A b-graphic sequence $\sigma=\left(s_{1}, \ldots, s_{n}\right)$ is potentially $\mathrm{A}_{\mathrm{l}, \mathrm{m}}^{\mathrm{b}}$-graphic if and only if $\sigma_{\mathrm{l}}$ is $\mathrm{b}$-graphic.

Proof. Assume that $\sigma$ is potentially $A_{l, m}^{b}$-graphic. Then $\sigma$ has a realization $\mathrm{G}$ with the vertex set $\mathrm{V}(\mathrm{G})=\left\{v_{1}\right.$, ldots, $\left.\nu_{n}\right\}$ such that $\mathrm{d}_{\mathrm{G}}\left(\nu_{\mathrm{i}}\right)=s_{\mathrm{i}}$ for $(1 \leq$ $i \leq n)$ and $G$ contains $J_{l, m}^{b}$ on the vertices $v_{1}, \ldots, v_{l+m}$, where $l+m \leq n$, so that $\mathrm{V}^{\mathrm{b}}\left(\mathrm{K}_{\mathrm{l}}\right)=\left\{v_{1}, \ldots, v_{\mathrm{l}}\right\}$ and $\mathrm{V}\left(\overline{\mathrm{K}}_{\mathrm{m}}^{\mathrm{b}}\right)=\left\{v_{\mathrm{l}+1}, \ldots, v_{\mathrm{l}+\mathrm{m}}\right\}$. We will show that by applying a sequence of $b$-exchanges to $G$ in order that there is one such realization $G^{\prime}$ such that $G^{\prime} \backslash v_{1}$ has degree sequence $\sigma_{1}$. If not, we may choose such a realization $\mathrm{H}$ of b-graphic sequence $\sigma$ such that the number of vertices adjacent to $v_{1}$ in $\left\{v_{l+m+1}, \ldots, v_{s_{1}+1}\right\}$ is maximum. Let $v_{i} \in\left\{v_{l+m+1}, \ldots, v_{s_{1}+1}\right\}$ 
and assume that there is no edge between $v_{1}$ and $v_{i}$ and let $v_{j} \in\left\{v_{s_{1}+2}, \ldots, v_{n}\right\}$ and there are $\mathrm{b}$ edges between $v_{1} v_{j}$. We may assume that $s_{i}>s_{j}$, since the order of $i$ and $j$ can be interchanged if $s_{i}<s_{j}$. Hence there is a vertex $v_{t}, t \neq i, j$ such that there are $\mathrm{b}$ edges between $v_{i}$ and $v_{t}$ and no edge between $v_{j}$ and $v_{\mathrm{t}}$. Clearly $\mathrm{G}=\left(\mathrm{H} \backslash\left\{v_{1}^{\mathrm{b}} v_{\mathrm{j}}, v_{i}^{\mathrm{b}} v_{\mathrm{t}}\right\}\right) \bigcup\left\{v_{1}^{\mathrm{b}} v_{i}, v_{j}^{\mathrm{b}} v_{\mathrm{t}}\right\}$-where $v_{i}^{\mathrm{b}} v_{\mathrm{j}}$ means that there are $\mathrm{b}$ edges between $v_{i}$ and $v_{j}$ is a realization of $\sigma$ such that $\mathrm{d}_{\mathrm{G}}\left(v_{i}\right)=s_{i}$ for $1 \leq i \leq n, G$ contains $S_{l, m}^{b}$ on $v_{1}, \ldots, v_{l+m}$ with $V^{b}\left(K_{l}\right)=\left\{v_{1}, \ldots, v_{l}\right\}$ and $\mathrm{V}\left(\overline{\mathrm{K}}_{\mathrm{m}}^{\mathrm{b}}\right)=\left\{v_{\mathrm{l+1}}, \ldots, v_{\mathrm{l}+\mathrm{m}}\right\}$ and $\mathrm{G}$ has the number of vertices adjacent to $v_{1}$ in $\left\{v_{l+m+1}, \ldots, v_{s_{1}+1}\right\}$ larger than that of $\mathrm{H}$. This contradicts the choice of $H$. Repeating this procedure, we can see that $\sigma_{i}$ is potentially $A_{l-i}^{b}$-graphic successively for $i=2, \ldots, l$. In particular, $\sigma_{l}$ is b-graphic.

Conversely suppose that $\sigma_{l}$ is b-graphic and is realized by a graph $G_{l}$ with a vertex set $V\left(G_{l}\right)=\left\{v_{l+1}, \ldots, v_{n}\right\}$ such that $d_{G_{l}}\left(v_{i}\right)=s_{i}$ for $l+1 \leq i \leq n$. For $i=l, \ldots, 1$ form $G_{i-1}$ from $G_{i}$ by adding a new vertex $v_{i}$ that is adjacent to each of $v_{i+1}, \ldots, v_{l+m}$ with $b$-edges and also to the vertices of $G_{i}$ with degrees $s_{l+m+1}^{i-1}-b, \ldots, s_{d_{i}+1}^{i-1}-b$. Then for each $i, G_{i}$ has degrees given by $\pi_{i}$ and $G_{i}$ contains $J_{l-i, m}^{b}$ on $l+m-i$ vertices $v_{i+1}, \ldots, v_{l+m}$ whose degrees are $s_{i+1}-$ $i b, \ldots, s_{l+m}-i b$ so that $V\left(K_{l-i}^{b}\right)=\left\{v_{i+1}, \ldots, v_{l}\right\}$ and $V\left(\bar{K}_{m}^{b}\right)=\left\{v_{l+1}, \ldots, v_{l+m}\right\}$. In particular, $G_{0}$ has degrees given by $\sigma$ and contains $S_{l, m}^{b}$ on $l+m$ vertices $v_{1}, \ldots, v_{l+m}$ whose degrees are $s_{1}, \ldots, s_{l+m}$ so that $V\left(K_{l}^{b}\right)=\left\{v_{1}, \ldots, v_{l}\right\}$ and $\mathrm{V}\left(\overline{\mathrm{K}}_{\mathrm{m}}^{\mathrm{b}}\right)=\left\{v_{\mathrm{l+1}}, \ldots, v_{\mathrm{l+m}}\right\}$. Hence the result follows.

Now we prove a sufficient condition for a b-graphic sequence to be potentially $A_{l}^{b}$-graphic.

Theorem 32 Let $\mathrm{n} \geq \mathrm{l}+\mathrm{m}$ and let $\sigma=\left(\mathrm{s}_{1}, \ldots, \mathrm{s}_{\mathrm{n}}\right)$ be a b-graphic sequence. If $\mathrm{s}_{\mathrm{l}+\mathrm{m}} \geq 2 \mathrm{bl}+\mathrm{bm}-2$, then $\sigma_{i}$ is potentially $\overline{\mathrm{A}}_{\mathrm{l}, \mathrm{m}}^{\mathrm{b}}$-graphic.

Proof. Let $n \geq l+m$ and let $\sigma=\left(s_{1}, \ldots, s_{n}\right)$ be a nonincreasing b-graphic sequence with $s_{l+m} \geq 2 b l+m-2$. By Theorem $17, \sigma$ is potentially $K_{l}^{b}$-graphic and hence by Lemma 33, $A_{l}^{b}$-graphic. Therefore, we may assume that $\mathrm{G}$ is a realization of $\sigma$ with a vertex set $V(G)=v_{1}, \ldots, v_{n}$ such that $d_{G}\left(v_{i}\right)=s_{i}$, $(1 \leq i \leq n)$ and $G$ contains $K_{l}^{b}$ on $v_{1}, \ldots, v_{l}$, that is, $V\left(K_{l}^{b}\right)=\left\{v_{1}, \ldots, v_{l}\right\}$ and $M=e_{G}\left(\left\{v_{1}, \ldots, v_{l}\right\},\left\{v_{l+1}, \ldots, v_{l+m}\right\}\right)$ (that is, the number of edges between $\left\{v_{1}, \ldots, v_{l}\right\}$ and $\left.\left\{v_{l+1}, \ldots, v_{l+m}\right\}\right)$ is maximum. If $M=b l m$, then $G$ contains $\bar{S}_{l, m}^{b}$ on $v_{1}, \ldots, v_{l+m}$ with $\mathrm{V}\left(\overline{\mathrm{K}}_{\mathrm{m}}^{\mathrm{r}}\right)=\left\{v_{\mathrm{l}+1}, \ldots, v_{\mathrm{l}+\mathrm{m}}\right\}$. In other-words, sigma is potentially $\bar{A}_{\mathrm{l}, \mathrm{m}}^{\mathrm{b}}$-graphic. Assume that $\mathrm{M}<\mathrm{blm}$. Then there exists a $v_{\mathrm{k}} \in$ $\left\{v_{1}, \ldots, v_{l}\right\}$ and $v_{m} \in\left\{v_{l+1}, \ldots, v_{l+m}\right\},(i \neq j)$ such that $e_{G}\left(v_{k}, v_{m}\right)<b$. Let

$$
A=N_{G \backslash\left\{v_{1}, \ldots, v_{l+m}\right\}}\left(v_{k}\right) \backslash N_{G \backslash\left\{v_{1}, \ldots, v_{l}\right\}}\left(v_{m}\right),
$$




$$
\mathrm{B}=\mathrm{N}_{\mathrm{G} \backslash\left\{v_{1}, \ldots, v_{\mathrm{l}+\mathrm{m}}\right\}}\left(v_{\mathrm{k}}\right) \cap \mathrm{N}_{\mathrm{G} \backslash\left\{v_{1}, \ldots, \nu_{\mathrm{l}}\right\}}\left(v_{\mathrm{m}}\right) .
$$

Then $e_{\mathrm{G}}(x, y)=\mathrm{b}$ for $x \in \mathrm{N}_{\mathrm{G} \backslash\left\{v_{1}, \ldots, v_{l}\right\}}\left(v_{\mathrm{m}}\right)$ and $\mathrm{y} \in \mathrm{N}_{\mathrm{G} \backslash\left\{v_{1}, \ldots, \nu_{l+m}\right\}}\left(v_{\mathrm{k}}\right)$. Otherwise, if $e_{G}(x, y)<b$, then $G^{\prime}=\left(G \backslash\left\{v y, v_{m} x\right\}\right) \cup\left\{v_{k} v_{m}, x y\right\}$ is a realization of $\pi$ and contains $\overline{\mathrm{J}}_{\mathrm{l}, \mathrm{m}}^{\mathrm{b}}$ on $v_{1}, \ldots, v_{\mathrm{l}+\mathrm{m}}$ with $\mathrm{V}\left(\mathrm{K}_{\mathrm{l}}^{\mathrm{b}}\right)=\left\{v_{1}, \ldots, v_{\mathrm{l}}\right\}$ and $\left(\overline{\mathrm{K}}_{\mathrm{m}}^{\mathrm{b}}\right)=\left\{v_{\mathrm{l}+1}, \ldots, v_{\mathrm{l}+\mathrm{m}}\right\}$ such that

$$
e_{G^{\prime}}\left(\left\{v_{1}, \ldots, v_{l}\right\},\left\{v_{l+1}, \ldots, v_{l+m}\right\}\right)>M,
$$

which contradicts the choice of G. Thus B is b-complete. We consider the following two cases.

Case 1. Let $A=\emptyset$. Then $2 b l+b m-2 \leq d_{k}=d_{G}\left(v_{k}\right)<b l+b m-1+b|B|$, and so $|B| \geq b l$. Since each vertex in $N_{G \backslash v_{1}, \ldots, \nu_{l}}\left(\nu_{m}\right)$ is adjacent to each vertex in $\mathrm{B}$ by $\mathrm{b}$ edges and $\left|\mathrm{N}_{\mathrm{G} \backslash\left\{v_{1}, \ldots, \nu_{\mathrm{l}}\right\}}\left(\nu_{\mathrm{m}}\right)\right| \geq 2 \mathrm{bl}+\mathrm{bm}-2=\mathrm{bl}+\mathrm{bm}-1$. It can be easily seen that the $b$ induced subgraph of $N_{G \backslash\left\{v_{1}, \ldots, v_{l}\right\}}\left(v_{m}\right) \cup\left\{v_{m}\right\}$ in $G$ contains $\bar{J}_{l, m}^{b}$ as a subgraph. Thus $\pi$ is potentially $\bar{A}_{l, m^{-}}^{b}$ graphic.

Case 2. Let $A \neq \emptyset$. Let $a \in A$. If there are $x, y \in N_{G \backslash\left\{v_{1}, \ldots, v_{l}\right\}}\left(v_{m}\right)$ such that $e_{G}(x, y)<b$ then $G^{\prime}=\left(G_{\backslash\left\{v_{m} x, v_{m} y, v_{k} a\right\}} \cup\left\{v_{k} v_{m}, a v_{m}, x y\right\}\right.$ is a realization of $\sigma$ and contains $\overline{\mathrm{J}}_{\mathrm{l}, \mathrm{m}}^{\mathrm{b}}$ on $v_{1}, \ldots, v_{\mathrm{l}+\mathrm{m}}$ with $\mathrm{V}\left(\mathrm{K}_{\mathrm{l}}^{\mathrm{b}}\right)=\left\{v_{1}, \ldots, v_{\mathrm{l}}\right\}$ and $\left(\overline{\mathrm{K}}_{\mathrm{m}}^{\mathrm{r}}\right)=\left\{v_{\mathrm{l}+1}, \ldots, v_{\mathrm{l}+\mathrm{m}}\right\}$ such that $\mathrm{e}_{\mathrm{G}^{\prime}}\left(\left\{v_{1}, \ldots, v_{\mathrm{l}}\right\},\left\{v_{\mathrm{l}+1}, \ldots, v_{\mathrm{l}+\mathrm{m}}\right\}\right)>\mathrm{M}$ which contradicts the choice of $\mathrm{G}$. Thus $\mathrm{N}_{\mathrm{G} \backslash\left\{v_{1}, \ldots, \nu_{l}\right\}}\left(v_{\mathrm{m}}\right)$ is b-complete. Since

$$
\left|\mathrm{N}_{\mathrm{G} \backslash\left\{v_{1}, \ldots, v_{\mathrm{l}}\right\}}\left(v_{\mathrm{m}}\right)\right| \geq \mathrm{bl}+\mathrm{bm}-1 \text { and } \mathrm{e}_{\mathrm{G}}\left(v_{\mathrm{m}}, z\right)=\mathrm{b}
$$

for any $z \in \mathrm{N}_{\mathrm{G} \backslash\left\{v_{1}, \cdots, v_{l}\right\}}\left(v_{\mathrm{m}}\right)$, it is easy to see that the induced b-subgraph of $\mathrm{N}_{\mathrm{G} \backslash\left\{v_{1}, \ldots, v_{v}\right\}}\left(v_{\mathrm{m}}\right) \cup\left\{v_{\mathrm{m}}\right\}$ in $\mathrm{G}$ is b-complete, and so contains $\overline{\mathrm{J}}_{l, \mathrm{~m}}^{\mathrm{b}}$ as a b-subgraph. Thus $\sigma$ is potentially $\bar{\AA}_{l, m}^{b}$-graphic.

\section{New results for split sequences}

Let $n \geq l+m$ and let $\sigma=\left(s_{1}, \ldots, s_{n}\right)$ be a nonincreasing sequence of nonnegative integers with $s_{l} \geq b(l+m)-1$ and $s_{l+m} \geq b l$. We define sequences $\sigma_{1}, \ldots, \sigma_{l}$ as follows. We first construct the sequence

$$
\sigma_{1}=\left(s_{2}-b, \ldots, s_{l}-b, s_{l+1}-b, \ldots, s_{l+m}-b, s_{l+m+1}^{1}, \ldots, s_{n}^{1}\right)
$$

from $\sigma$ by reducing 1 the largest term that has not already been reduced $b$ times, and then reordering the last $n-l-m$ terms to be nonincreasing. For $2 \leq i \leq b$, we construct

$$
\sigma_{i}=\left(s_{i+1}-i b, \ldots, s_{l}-i b, s_{l+1}-b r, \ldots, s_{l+m}-i b, s_{l+m+1}^{i}, \ldots, s_{n}^{i}\right)
$$


from

$$
\begin{aligned}
\sigma_{i-1}= & \left(s_{i}-(i-1) b, \ldots, s_{l}-(i-1) b, s_{l+1}-(i-1) b, \ldots,\right. \\
& \left.s_{l+m}-(i-1) b, s_{l+m+1}^{i-1}, \ldots, s_{n}^{i-1}\right)
\end{aligned}
$$

by deleting $s_{i}-(i-1) b$, reducing the first $s_{i}-(i-1) b$ remaining terms of $s_{i-1}$ by one that has not already been reduced $b$ times, and then reordering the last $n-l-m$ terms to be nonincreasing.

We start with the following lemma.

Lemma 33 A nonincreasing integer sequence $\sigma=\left(s_{1}, \ldots, s_{n}\right)$ is potentially $\mathrm{A}_{\mathrm{l}, \mathrm{m}}^{\mathrm{b}}$-graphic if and only if it is potentially $\mathrm{J}_{\mathrm{l}, \mathrm{m}}^{\mathrm{b}}$-graphic.

Proof. We only need to prove that if $\sigma=\left(s_{1}, \ldots, s_{n}\right)$ is potentially $J_{l, m^{-}}^{b}$ graphic, then it is potentially $A_{l, m}^{b}$-graphic. We may choose a realization $G$ of $\sigma$ with vertex set $\mathrm{V}(\mathrm{G})=\left\{v_{1}, \ldots, v_{n}\right\}$ such that $\mathrm{d}_{\mathrm{G}}\left(v_{i}\right)=s_{i}$ for $1 \leq i \leq$ $\mathrm{n}$, the induced b-subgraph $\mathrm{G}\left[\left\{v_{1}, \ldots, v_{l+\mathrm{m}}\right\}\right]$ of $\left\{v_{1}, \ldots, v_{l+m}\right\}$ in $\mathrm{G}$ contains $J_{l, m}^{b}$ as its b-subgraph and $\left|\mathrm{V}\left(\mathrm{K}_{l}^{\mathrm{b}}\right) \cap\left\{v_{1}, \ldots, v_{l}\right\}\right|$ is maximum. Denote $\mathrm{H}=$ $\mathrm{G}\left[\left\{v_{1}, \ldots, v_{l+m}\right\}\right]$. If $\left|\mathrm{V}\left(\mathrm{K}_{\mathrm{l}}^{\mathrm{b}}\right) \cap\left\{v_{1}, \ldots, \mathrm{d}_{l}\right\}\right|=l$, that is, $\mathrm{V}\left(\mathrm{K}_{\mathrm{l}}^{\mathrm{b}}\right)=\left\{v_{1}, \ldots, v_{l}\right\}$, then $\sigma$ is potentially $A_{l, m}^{b}$-graphic. Assume that $\left|\mathrm{V}\left(\mathrm{K}_{l}^{\mathrm{b}}\right) \cap\left\{v_{1}, \ldots, v_{l}\right\}\right|<l$. Then there exists $v_{i} \in\left\{v_{1}, \ldots, v_{l}\right\} \backslash \mathrm{V}\left(\mathrm{K}_{\mathrm{l}}^{\mathrm{b}}\right)$ and a $v_{j} \in \mathrm{V}\left(\mathrm{K}_{\mathrm{l}}^{\mathrm{b}}\right) \backslash\left\{v_{1}, \ldots, v_{\mathrm{l}}\right\}$. Let $A=N_{H}\left(v_{j}\right) \backslash\left(\left\{v_{i}\right\} \cup N_{H}\left(v_{i}\right)\right)$ and $B=N_{G}\left(v_{i}\right) \backslash\left(\left\{v_{j}\right\} \cup N_{G}\left(v_{j}\right)\right)$. Since $\mathrm{d}_{\mathrm{G}}\left(v_{i}\right) \geq \mathrm{d}_{\mathrm{G}}\left(v_{j}\right)$. We have $|\mathrm{B}| \geq|\mathrm{A}|$. Let us choose any subset $\mathrm{C} \subseteq \mathrm{B}$ such that $|C|=|A|$. Now form a new realization $G^{\prime}$ of $s$ by a sequence of $b$-exchanges the $b$-edges of the star centralized at $v_{j}$ with end vertices in $A$ with the non b-edges of the star centralized at $v_{j}$ with end vertices in $C$, and by a sequence of $b$-exchange the b-edges of the star centralized at $v_{i}$ with end vertices in $C$ with the non $b$-edges of the star centralized at $v_{i}$ with end vertices in $A$. It is easy to see that $\mathrm{G}^{\prime}$ contains $\mathrm{J}_{l, m}^{\mathrm{b}}$ on $\left\{v_{1}, \ldots v_{l+m}\right\}$ so that $\left|\mathrm{V}\left(\mathrm{K}_{l}^{\mathrm{b}}\right) \cap\left\{v_{1}, \ldots, v_{l}\right\}\right|$ is larger than that of $\mathrm{G}$, which contradicts to the choice of $\mathrm{G}$.

In the next result, we use the result of Fulkerson et al. [24] and prove a necessary and sufficient condition for a b-graphic sequence $s$ to be potentially $\mathrm{J}_{l, \mathrm{~m}}^{\mathrm{b}}$-graphic.

Theorem 34 Let $\mathrm{n} \geq \mathrm{l}+\mathrm{m}$ and $\sigma=\left(\mathrm{s}_{1}, \ldots, \mathrm{s}_{\mathrm{n}}\right)$ be a nonincreasing even sequence of nonnegative integers, where $s_{l} \geq b(l+m-1)$ and $s_{l+m} \geq l b$. 
Then $\sigma$ is potentially $\mathrm{J}_{\mathrm{l}, \mathrm{m}}^{\mathrm{b}}$-graphic if and only if

$$
\begin{aligned}
& \sum_{i=1}^{p}\left(s_{i}-b(l+m-1)\right)+\sum_{i=b+1}^{l+p^{\prime}}\left(s_{i}-b l\right)+\sum_{i=l+m+1}^{l+m+q} s_{i} \leq \\
& r\left(p+p^{\prime}+q\right)\left(p+p^{\prime}+q-1\right)-r p(p-1)-2 b p p^{\prime} \\
& +\sum_{i=p+1}^{r} \min \left\{b q, s_{i}-b(l+m-1)\right\} \\
& +\sum_{i=l+p^{\prime}+1}^{l+m} \min \left\{b\left(p^{\prime}+q\right), s_{i}-b l\right\}+\sum_{i=l+m+q+1}^{n} \min \left\{b\left(p+p^{\prime}+q\right), s_{i}\right\}
\end{aligned}
$$

for any $1 \leq \mathrm{l} \leq \mathrm{n}, 1 \leq \mathrm{m} \leq \mathrm{n}$, for any $\mathrm{p}$, $\mathrm{p}^{\prime}$ with $0 \leq \mathrm{p} \leq \mathrm{l}, 0 \leq \mathrm{p}^{\prime} \leq \mathrm{m}$ and $0 \leq \mathrm{q} \leq \mathrm{n}-\mathrm{l}-\mathrm{m}$.

Proof. To prove the necessity, by Lemma 33, let $G$ be a graph with vertex set $V(G)=\left\{v_{1}, \ldots, v_{n}\right\}$ such that $d_{G}\left(v_{i}\right)=s_{i}$ for $1 \leq i \leq n$ and $G$ contains $\mathrm{J}_{\mathrm{l}, \mathrm{m}}^{\mathrm{b}}$ on $v_{1}, \ldots, v_{\mathrm{l}+\mathrm{m}}$ with $\mathrm{V}\left(\mathrm{K}_{\mathrm{l}}^{\mathrm{b}}\right)=\left\{v_{1}, \ldots, v_{\mathrm{l}}\right\}$ and $\mathrm{V}\left(\overline{\mathrm{K}}_{\mathrm{m}}^{\mathrm{b}}\right)=\left\{v_{\mathrm{l}+1}, \ldots, v_{\mathrm{l}+\mathrm{m}}\right\}$. The removal of the $\mathrm{b}$ edges induced by $\left\{v_{1}, \ldots, v_{l}\right\}$ and the $\mathrm{b}$-edges between $\left\{v_{1}, \ldots, v_{l}\right\}$ and $\left\{v_{l+1}, \ldots, v_{l+m}\right\}$ results in a graph $G^{\prime}$ in which all degrees in $\left\{v_{1}, \ldots, v_{l}\right\}$ are reduced by $b(l+m-1)$ and all degrees in $\left\{v_{l+1}, \ldots, v_{l+m}\right\}$ are reduced by $l b$. For $0 \leq p \leq l, 0 \leq p^{\prime} \leq m$ and $0 \leq q \leq n-l-m$, denote $\mathrm{P}=\left\{v_{i} \mid 1 \leq i \leq p\right\}, \mathrm{P}^{\prime}=\left\{v_{i} \mid l+1\right.$ leq $\left.i \leq l+p^{\prime}\right\}, R=\left\{v_{i} \mid p+1 \leq i \leq\right.$ $l\}, R^{\prime}=\left\{v_{i} \mid l+p^{\prime}+1 \leq i \leq l+m\right\}, Q=\left\{v_{i} \mid l+m+1 \leq i \leq q+l+m\right\}$ and $S=\left\{v_{i} \mid q+l+m+1 \leq i \leq n\right\}$. The degree sum in the $b$-subgraph induced by $\mathrm{P} \cup \mathrm{P}^{\prime} \cup \mathrm{Q}$ is at most $\mathrm{b}\left(\mathrm{p}+\mathrm{p}^{\prime}+\mathrm{q}\right)\left(\mathrm{p}+\mathrm{p}^{\prime}+\mathrm{q}-1\right)-\mathrm{bp}(\mathrm{p}-1)-2 b p p^{\prime}$. Therefore,

$$
\begin{aligned}
& m=\sum_{i=1}^{p}\left(s_{i}-b(l+m-1)\right)+\sum_{i=r+1}^{b+p^{\prime}}\left(s_{i}-b l\right)+\sum_{i=l+m+1}^{l+m+q} s_{i} \\
& -b\left(p+p^{\prime}+q\right)\left(p+p^{\prime}+q-1\right)-b p(p-1)-2 b p p^{\prime}
\end{aligned}
$$

is the minimum number of edges of $G^{\prime}$ with exactly one end vertex in $P \cup P^{\prime} \cup Q$. On the other hand, the maximum number of edges of $G^{\prime}$ with exactly one end 
vertex in $R \cup R^{\prime} \cup S$ is

$$
\begin{aligned}
& M=\sum_{i=p+1}^{b} \min \left\{b q, s_{i}-b(l+m-1)\right\}+\sum_{i=b+p^{\prime}+1}^{l+m} \min \left\{b\left(p^{\prime}+q\right), s_{i}-b l\right\} \\
& +\sum_{i=l+m+q+1}^{n} \min \left\{b\left(p+p^{\prime}+q\right), s_{i}\right\}
\end{aligned}
$$

We observe that in the graph $G^{\prime}, m \leq M$ is true. This proves the necessity.

To prove the sufficiency, we shall use the following well-known result of Fulkerson et al. [24]. Let $H$ be a b-graph on the vertex set $V(H)=\left\{v_{1}, \ldots, v_{n}\right\}$. There exists a $b$-subgraph $G \subseteq H$ such that every vertex $v_{i}$ has degree $s_{i}$, if and only if

$$
\sum_{i=1}^{n} s_{i} \text { is even, }
$$

and for every $A, B \subseteq V(H)$ such that $A \cap B=s$, we have

$$
\sum_{v_{i} \in A} s_{i} \leq \sum_{v_{i} \in A, v_{j} \in V(H) \backslash B} e_{H}\left(v_{i}, v_{j}\right)+\sum_{v_{i} \in B} s_{i} .
$$

We now continue to proceed with the proof of sufficiency. Let $n \geq l+m$ and $\sigma=\left(s_{1}, \ldots, s_{n}\right)$ be a nonicreasing sequence of nonnegative integers, where $s_{l} \geq l+m-1, s_{l+m} \geq l$ and $\sum_{i=1}^{n} s_{i}$ is even. Let $s^{\prime}=\left(s_{1}^{\prime}, \ldots, s_{n}^{\prime}\right)$, where $s_{i}^{\prime}=s_{i}-l-m+1$ for $1 \leq i \leq l, s_{i}^{\prime}=s_{i}-b$ for $l+1 \leq i \leq l+m$ and $s_{i}^{\prime}=s_{i}$ for $l+m+1 \leq i \leq n$. Let $H$ be the graph obtained from $K_{n}^{b}$ with vertex set $\mathrm{V}\left(\mathrm{K}_{\mathrm{n}}^{\mathrm{b}}\right)=\left\{v_{1}, \ldots, v_{n}\right\}$ by deleting all edges of the complete $\mathrm{b}$-subgraph induced by $\left\{v_{1}, \ldots, v_{l}\right\}$ and all edges between $\left\{v_{1}, \ldots, v_{l}\right\}$ and $\left\{v_{1+1}, \ldots, v_{l+m}\right\}$. It is easy to see that $s$ is potentially $A_{l, m}^{b}$-graphic if and only if $H$ has a subgraph $G$ with the degree sequence $s^{\prime}$ such that every vertex $v_{i}$ has degree $s_{i}^{\prime}$. Observe that between two disjoint odd cycles of $\mathrm{H}$ there is an edge. Therefore, $\mathrm{H}$ satisfies the odd-cycle condition and we apply (14) and (15).

Let $\mathrm{K}=\left\{v_{1}, \ldots, v_{\mathrm{l}}\right\}, \mathrm{K}^{\prime}=\left\{v_{\mathrm{l}+1}, \ldots, v_{\mathrm{l}+\mathrm{m}}\right\}$ and $\mathrm{A}, \mathrm{B} \subseteq \mathrm{V}(\mathrm{H})$ such that $\mathrm{A} \cap \mathrm{B}=$ s. Let $A_{1}=A \cap K, A_{1}^{\prime}=A \cap K^{\prime}, A_{2}=A \backslash\left(K \cup K^{\prime}\right), B_{1}=B \cap K, B_{1}^{\prime}=B \cap K, B_{2}=$ $B \backslash\left(K \cup K^{\prime}\right)$ and set $p=\left|A_{1}\right|, p^{\prime}=\left|A_{1}^{\prime}\right|, q=\left|A_{2}\right|, b_{1}=\left|B_{1}\right|, b_{1}^{\prime}=\left|B_{1}^{\prime}\right|, b_{2}=\left|B_{2}\right|$. For convenience, we denote

$$
L\left(p, p^{\prime}, q\right)=\sum_{i=1}^{p}\left(s_{i}-b(l+m-1)\right)+\sum_{i=r+1}^{r+p^{\prime}}\left(s_{i}-b l\right)+\sum_{i=r+s+1}^{r+s+q} s_{i},
$$




$$
\begin{aligned}
& R\left(p, p^{\prime}, q\right)=b\left(p+p^{\prime}+q\right)\left(p+p^{\prime}+q-1\right)-b p(p-1)-2 b p p^{\prime} \\
&+\sum_{i=p+1}^{b} \min \left\{b q, s_{i}-b(l+m-1)\right\}+\sum_{i=r+p^{\prime}+1}^{l+m} \min \left\{b\left(p^{\prime}+q\right), s_{i}-b l\right\} \\
&+\sum_{i=l+m+q+1}^{n} \min \left\{b\left(p+p^{\prime}+q\right), s_{i}\right\}, \\
& L^{\prime}(A, B)=\sum_{v_{i} \in A} s_{i}^{\prime}=\sum_{v_{i} \in A_{1}}\left\{s_{i}-b(l+m-1)\right\}+\sum_{v_{i} \in A_{1}^{\prime}}\left\{s_{i}-b l\right\}+\sum_{v_{i} \in A_{2}} s_{i}, \\
& R^{\prime}(A, B)= \sum_{v_{i} \in A, v_{j} \in V(H) \backslash B} e_{H}\left(v_{i}, v_{j}\right)+\sum_{v_{i} \in B} s_{i}^{\prime} \\
& \sum_{v_{i} \in A, v_{j} \in V(H) \backslash B} e_{H}\left(v_{i}, v_{j}\right)+\sum_{v_{i} \in B_{1}}\left(s_{i}-b(l+m-1)\right)+\sum_{v_{i} \in B_{1}^{\prime}}\left(s_{i}-l b\right)+\sum_{v_{i} \in B_{2}} s_{i} .
\end{aligned}
$$

Clearly, $\mathrm{L}^{\prime}(A, \mathrm{~B}) \leq \mathrm{L}\left(\mathrm{p}, \mathrm{p}^{\prime}, \mathrm{q}\right)$. Further $\sum_{v_{i} \in A, v_{j} \in \mathrm{V}(\mathrm{H}) \backslash \mathrm{B}} e_{\mathrm{H}}\left(v_{i}, v_{j}\right)$ is the number of counting the edges of $H$ between $A$ and $V(H) \backslash(A \cup B)$ and double counting the edges induced by $A$. Thus we get

$$
\begin{aligned}
& \quad \sum_{v_{i} \in A, v_{j} \in V(H) \backslash B} e_{H}\left(v_{i}, v_{j}\right) \\
& =r\left(p+p^{\prime}+q\right)\left(p+p^{\prime}+q-1\right)-b p(p-1)-2 b p p^{\prime}+q b\left(l-p-b_{1}\right) \\
& +b\left(p^{\prime}+q\right)\left(m-p^{\prime}-b_{1}^{\prime}\right)+b\left(p+p^{\prime}+q\right)\left(n-l-m-q-b_{2}\right) \\
& =b\left(p+p^{\prime}+q\right)\left(p+p^{\prime}+q-1\right)-b p(p-1)-2 b p p^{\prime}+\sum_{i=p+1}^{l-b_{1}} q \\
& +\sum_{i=l+p^{\prime}+1}^{l+m-b_{1}^{\prime}}\left(p^{\prime}+q\right)+\sum_{i=l+m+q+1}^{n-b_{2}}\left(p+p^{\prime}+q\right) .
\end{aligned}
$$


Therefore,

$$
\begin{aligned}
& \mathrm{R}^{\prime}(\mathrm{A}, \mathrm{B})=\sum_{v_{\mathrm{i}} \in \mathrm{A}, v_{j} \in \mathrm{V}(\mathrm{H}) \backslash \mathrm{B}} e_{\mathrm{H}}\left(v_{i}, v_{j}\right)+\sum_{v_{i} \in \mathrm{B}_{1}}\left(s_{i}-\mathrm{b}(\mathrm{l}+\mathrm{m}-1)\right) \\
& +\sum_{v_{i} \in B_{1}^{\prime}}\left(s_{i}-l b\right)+\sum_{v_{i} \in B_{2}} s_{i} \\
& \geq b\left(p+p^{\prime}+q\right)\left(p+p^{\prime}+q-1\right)-b p(p-1)-2 b p p^{\prime}+\sum_{i=p+1}^{l-b_{1}} q \\
& +\sum_{i=l+p^{\prime}+1}^{l+m-b_{1}^{\prime}}\left(p^{\prime}+q\right)+\sum_{i=l+m+q+1+1}^{n-b_{2}}\left(p+p^{\prime}+q\right) \\
& +\sum_{i=l-b_{1}+1}^{l}\left(s_{i}-b(l+m-1)\right)+\sum_{i=l+m-b_{1}^{\prime}+1}^{l+m}\left(s_{i}-b r\right)+\sum_{i=n-b_{2}+1}^{n} s_{i} \\
& \geq r\left(p+p^{\prime}+q\right)\left(p+p^{\prime}+q-1\right)-b p(p-1)-2 b p p^{\prime} \\
& +\sum_{i=p+1}^{l} \min \left\{b q, s_{i}-b(l+m-1)\right\}+\sum_{i=l+p^{\prime}+1}^{l+m} \min \left\{b\left(p^{\prime}+q\right), s_{i}-b l\right\} \\
& +\sum_{i=l+m+q+1}^{n} \min \left\{r\left(p+p^{\prime}+q\right), s_{i}\right\} \\
& =R\left(p, p^{\prime}, q\right) \text {. }
\end{aligned}
$$

It follows from $L\left(p, p^{\prime}, q\right) \leq R\left(p, p^{\prime}, q\right)$ that $L^{\prime}(A, B) \leq R^{\prime}(A, B)$. By (14) and (15) $\mathrm{H}$ is a $b$-subgraph $\mathrm{G}$ with the degree sequence $s^{\prime}$ such that every vertex $v_{i}$ has degree $s_{i}^{\prime}$. Hence $s$ is potentially $A_{l, m}^{b}$-graphic. Thus the sufficiency is proved.

It is easy to enumerate the $\mathrm{J}_{l, m}$-split graphs on $\mathrm{n}$ vertices.

Theorem 35 If $\mathrm{l} \geq 0, \mathrm{~m} \geq 1, \mathrm{l}+\mathrm{m} \geq 1$, and $\mathrm{b} \geq 1$, then

1. there are $\lambda(\mathrm{b}, \mathrm{l}, \mathrm{m})=\left(\begin{array}{c}\mathrm{l}+\mathrm{m} \\ \mathrm{l}\end{array}\right)$ labeled $\mathrm{J}_{\mathrm{l}, \mathrm{m}}^{\mathrm{b}}$ and they are isomorphic;

2. there are $\beta(\mathrm{b}, \mathrm{l}, \mathrm{m})=\mathrm{l}+\mathrm{m}$ nonisomorphic $\mathrm{J}_{\mathrm{l}, \mathrm{m}}^{\mathrm{b}}$.

\section{Proof.}

1. Since $J_{l, m}$ has $l+m$ vertices, therefore there are $\left(\begin{array}{c}l+m \\ l\end{array}\right)$ ways to choose the vertices of $K_{l}$. If we consider two different labeled $J_{l, m}$ jsplit graphs, then 
the vertices of the clique parts correspond to each other, and the independent vertices of these graphs also correspond to each other, therefore these graphs are isomorphic.

2. Formally $J_{0, l+m}^{b}, J_{1, l+m-1}^{b}, \ldots, J_{0, l+m}^{b}$ are $l+m+1$ different possibilities, but the last two split graphs are isomorphic, therefore $\beta(l, m)=l+m$.

We can remark, that if $m \geq 1$, then $J_{l, m}$ is also a $J_{l+1, m-1}$ split graph.

\section{Known algorithms for graphic sequences}

In this section at first we present the classical Havel-Hakimi $(\mathrm{HH})$ algorithm, then its testing version (HHL), which even in the worts case in $\mathrm{O}(\boldsymbol{n})$ time decides whether an integer sequence is $(0,1, n)$-graphic. Then we describe algorithm HaVEL-HAKIMI-pqlm-SPLIT which in $\mathrm{O}(\mathrm{n})$ time decides the similar

problem for potentially $J_{l, m}^{(p, q)}$-graphic sequences, further a Havel-Hakimi type algorithm for recognition of $(a, b, n)$.

\subsection{HAVEL-HAKIMI algorithm (HH)}

If $n=1$, then there exists one $(0,1, n)$-graphic sequence: $(0)$. If $n \geq 2$, then Havel-Hakimi theorem (Theorem 9 ) gives a necessary and sufficient condition.

Input. $\mathrm{n}$ : the length of the sequence $s(\mathrm{n} \geq 2)$;

$\sigma=\left(s_{1}, \ldots, s_{n}\right)$ : the investigated $n$-regular sequence.

Output. L: logical variable ( $\mathrm{L}=0$ signalizes, that $\sigma$ is not graphic, while $\mathrm{L}=1$ means, that $\sigma$ is $(0,1, \mathrm{n})$-graphic) .

Working variable. i: cycle variables.

$\operatorname{HAVEL-HAKIMI}(n, \sigma)$

$01 \mathrm{~L}=0$

02 for $i=1$ to $n-1$

03 if $s_{s_{i}+i}==0$

04 return $\mathrm{L}$

05 for $j=i+1$ to $s_{i}+i$

$06 \quad s_{j}=s_{j}-1$

$07 \quad$ sort $\left(s_{i+1}, \ldots, s_{n}\right)$ in decreasing order

$08 \mathrm{~L}=1$

// line 01-07: test of the elements of $s$

09 return $\mathrm{L}$

// lines 03-04: s is not graphic

// lines 08-09: $s$ is graphic 


\subsection{Havel-Hakimi-Testing-Linear algorithm (HHTL)}

The original Havel-Hakimi algorithm in worst case requires quadratic time to test the $(0,1, n)$-regular sequences. Using the concepts weight point, reserve and cutting point we reduced the worst running time to $\mathrm{O}(\mathrm{n})$.

The definition of the weight point $w_{i}$ belonging to $s_{i}$ was introduced in [48] in connection with ERDös-Gallai-LinEAR and it is as follows. $w_{i}$ is the largest $k(1 \leq k \leq n)$ having the property $s_{k} \geq i$. But if $s_{1}<i$, then $w_{i}=0$. EGL exploits the property $\boldsymbol{w}_{i}$ ensuring that if $i \leq \boldsymbol{w}_{i}$, then the key expression $\min j, s_{k}$ in the Erdős-Gallai theorem equals to $i$, otherwise equals to $s_{k}$.

Here we extend the definition to be applicable also in the proof of the linearity of Chungphaisan-Erdös-Gallai. Now let $w_{i}$ the largest $k(1 \leq k \leq n)$ having the property $s_{k} \geq$ bi. But if $s_{1}<$ bi, then let $w_{i}=0$. In the case $b=1$ the new definition results the old one.

In HHL the weight point $w_{i}$ determines the increment of the tail capacity when we switch to the investigation of the next element of $\sigma$.

The remainder $r_{i}$ belonging to $s_{i}$ is defined as the unused part of the actual tail capacity and can be computed by the formulas

$$
r_{i}=w_{1}-1-s_{1}
$$

and

$$
r_{i}=w_{i}-r_{i-1}-s_{i} \quad \text { for } 1 \leq i \leq n-1 .
$$

The cutting point $y_{i}$ belonging to $s_{i}$ is $\max \left(i, w_{i}\right)$.

The programs of this paper are written using the pseudocode conventions descibed in [16].

Input. $\mathrm{n}$ : number of vertices $(\mathrm{n} \geq 1)$;

$\sigma=\left(s_{1}, \ldots, s_{n}\right):$ the investigated $n$-graphic sequence.

Output. L: logical variable.

Work variables. $i$ : cycle variable;

$r=\left(r_{1}, \ldots, r_{n}\right): r_{i}$ the reserve belonging to $s_{i}$;

$w=\left(w_{1}, \ldots, w_{n}\right): w_{i}$ the weight point belonging to $s_{i}$;

$H=\left(H_{1}, \ldots, H_{n}\right): H_{i}$ is the sum of the first $i$ elements of $s$.

Havel-Hakimi-Testing-Linear $(n, s)$

$01 \mathrm{~L}=0 \quad$ // lines 01: set the probable value

02 if $s_{1}==0 \quad / /$ lines $02-04$ : test of the sequence consisting of only zeros

$03 \mathrm{~L}=1$

04 return $\mathrm{L}$ 


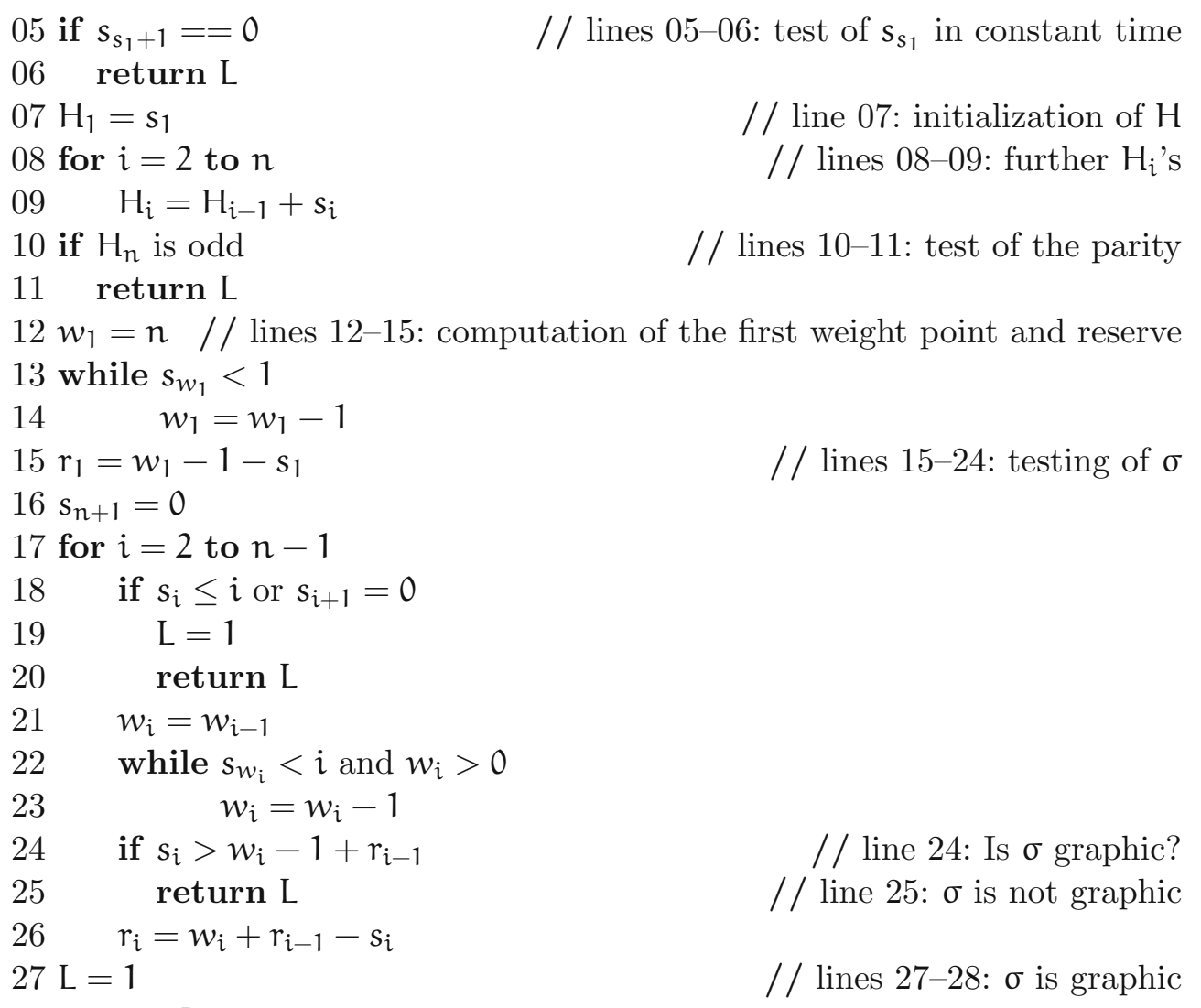
28 return $\mathrm{L}$

Theorem 36 The running time of HAVEL-HAKIMI-TESTING-LINEAR is in best case $\Theta(1)$, and in worst case is $\Theta(n)$.

Proof. If the condition in line 2 holds, then the running time is $\Theta(1)$. If not, then we reduce the actual $w$ at most $n$ times and the remaining operations require $\mathrm{O}(1)$ operations for all reductions.

\subsection{Erdős-Gallai-Chungphaisan-Linear algorithm (EGChL)}

The following algorithm tests the potential degree sequences of $(0, b, n)$-graphs. It is based on Theorem 13 .

Input. $\mathrm{n}$ : number of vertices $(\mathrm{n} \geq 1)$; $\sigma=\left(s_{1}, \ldots, s_{n}\right):$ a $(0, b, n)$-regular sequence;

$\mathrm{b}$ : the maximal permitted number of arcs between two vertices. 
Output. 1 or $0: 1$, if $s$ is $(0, b, n)$-graphic and 0 otherwise.

Work variable. $i$ : cycle variable;

$r=\left(r_{1}, \ldots, r_{n}\right): r_{i}$ is the reserve belonging to $s_{i}$;

$w=\left(w_{1}, \ldots, w_{n}\right): w_{i}$ is the weightpoint belonging to $s_{i}$.

\section{ERDŐS-GALlai-ChungPhaisan-Linear $(n, \sigma, b)$}

$01 \mathrm{H}_{1}=\mathrm{s}_{1}$

// line 01: initialization of $\mathrm{H}_{1}$

02 for $i=2$ to $n-1 \quad / /$ line 02-03: computation of the elements of $H$

$03 \quad \mathrm{H}_{\mathrm{i}}=\mathrm{H}_{\mathrm{i}-1}+\mathrm{s}_{\mathrm{i}}$

04 if $\mathrm{H}_{n}$ is odd

// line 04-05: test of the parity

05 return 0

$06 w=\mathrm{n}$

07 for $i=1$ to $n-1$

// lines 06: initialization of the first weight point

08

while $s_{w}<i b$ and $w>0$

// lines 07-12: test of $\sigma$

09

$w=w-1$

$10 y=\max (i, w)$

11 if $\mathrm{H}_{\mathrm{i}}>\mathrm{bi}(\mathrm{y}-1)+\mathrm{H}_{\mathrm{n}}-\mathrm{H}_{\mathrm{y}}$

12 return 0

13 return 1

// line 13: acceptance of $\sigma$

Theorem 37 The running time of ERDös-Gallai-ChungPhaisan-LinEAR is $\Theta(\mathrm{n})$ in all cases.

Proof. Lines $01-05$ require $\Theta(n)$ time. Since the value of $w$ is strictly decreasing, lines $06-13$ require $\mathrm{O}(\mathrm{n})$ time, therefore the running time is $\Theta(n)$ in all cases.

Let us consider two examples. Let $\mathrm{b}=3$ and $\sigma^{\prime}=(13,10,5,5,4,1) . \mathrm{H}_{6}=38$ is even. If $i=1$, then $w_{i}=y=5$ and the condition in line 11 is not satisfied $(13 \leq 3 \cdot 1 \cdot(5-1))$. If $i=2$, then $w_{i}=y=2$ and the condition in line 11 holds $(23>3 \cdot 2 \cdot(2-1))+5+5+4+1$, therefore $\sigma$ is not $(0,3,6)$-graphic.

Let $\mathrm{b}$ remain 3 , but change $\sigma$ to $\sigma^{\prime}=(13,10,5,5,4,3)$. The first difference comparing with the previous example comes when $i=2$. Now $23 \leq 3 \cdot 2 \cdot(2-$ 1) $)+5+5+4+3$, and the condition in line 11 holds for $i=3,4$ and 5 too, therefore $\sigma^{\prime}$ is $(0,3,6)$-graphic.

Using Corollary 15 it is easy to test an $(\mathrm{a}, \mathrm{b}, \mathrm{n})$-regular sequence $\sigma$ whether it is $(a, b, n)$-graphic. We use EGCHL with input sequence $\sigma^{\prime}=\left(s_{1}-a(n-\right.$ $\left.1), \ldots, s_{n}-a(n-1)\right)$. 


\section{Known algorithms for split sequences}

In this section we describe the linear time algorithm proposed for the recognition and reconstruction of potentially psplit and jsplit sequences.

\subsection{HAMMER-SIMEONE-PSPLIT algorithm (HSPS)}

The following algorithms was proposed in 1981 by Hammer and Simeone [34]. Ist base is Theorem 26 .

Let $G$ be a graph with degree sequence $d=\left(d_{1}, \ldots, d_{n}\right)$.

Input. $\mathrm{n}$ : number of elements of $\delta$;

$\delta=\left(d_{1}, \ldots, d_{n}\right)$ : a graphic sequence.

Output. 1 or $0: 1$, if $\mathrm{d}$ is potentially psplit sequence.

Work variable. $i, \mathrm{k}$ : cycle variables;

$\Sigma_{1}, \Sigma_{2}$ : actual sums of the degrees.

HAMmER-SimEONE-LineAR $(n, \delta)$

$01 \mathrm{k}=0$

$02 \Sigma_{1}=0$

03 while $d_{k+1} \geq k-1$ and $k<n$

$04 \quad \mathrm{~m}=\mathrm{k}+1$

$05 \quad \Sigma_{1}=\Sigma_{1}+\mathrm{d}_{\mathrm{k}}$

$06 \quad k=k+1$

$07 \Sigma_{2}=\mathrm{m}(\mathrm{m}-1)$

08 for $i=m+1$ to $n$

$09 \quad \Sigma_{2}=\Sigma_{2}+\mathrm{d}_{\mathrm{i}}$

10 if $\Sigma_{1} \neq \Sigma_{2}$

11 return 0

12 return $\mathrm{G}$ i'is psplit, maximal clique size is $\mathrm{m} / /$ line 12: $\mathrm{G}$ is psplit graph

Theorem 38 Let $\mathrm{G}$ a graph with degree sequence $\delta$. Algorithm HammerSIMEONE-LINEAR decides, if $\mathrm{G}$ is a psplit graph and computes the maximal clique size in $\Theta(\mathrm{n})$ time.

Proof. Lines 01-02 require $\mathrm{O}(1)$ time, lines 03-09 $\Theta(\mathrm{n})$ time and lines 10-12 $\mathrm{O}(1)$ time.

\subsection{Further linear algorithms for psplit sequences}

In 1980 Golumbic [26], in 2003 Feder et al. [20], in 2007 Heggernes and Kratsch [37] proposed linear time algorithm for the recognition of psplit graphs. 


\subsection{Havel-Hakimi-Testing-JSplit algorithm (HHJST)}

In 2012 Yin [87] described HHJST, a Havel-Hakimi type linear algorithm for the recognition of potentially jsplit sequences.

\section{New algorithms}

In this section we present two simple algorithms, which decide whether a sequence of nonnegative integers is $A_{l}^{b}$-graphic or $J_{l, m}^{b}$-graphic, and if the answer is yes, then they compute the maximal suitable $l$ too.

These algorithms require in worst case only $O(n)$ time even for $(a, b, n)$ regular input, and are quicker for $(a, b, n)$-graphic input., since then the sorting can be omitted.

We remark, that earlier only for pseudo-split graphs was published a linear time testing algoritm [58].

\subsection{Algorithm Ab-l-MaX}

For given sequence $\sigma=\left(s_{1}, \ldots, s_{n}\right)$ of nonnegative integers and given nonnegative integer $b$ algorithm A-b-l-MAx computes the maximal $l$ for which the sequence $s$ is $A_{1}^{b}$-graphic.

Input. $\mathrm{n} \geq 1$ : the length of the sequence $s$;

$\sigma=\left(s_{1}, \ldots, s_{n}\right):$ a sequence of nonnegative integers;

$\mathrm{b}$ : the maximal permitted number of arcs between two different vertices.

Output. l: the maximal value for which $d$ is $A_{l}^{b}$-graphic.

Work variable. i: cycle variable.

A-b-l-MAx $(n, \sigma, b)$

01 Counting-Sort $(n, \sigma)$

$02 l=1$

03 while $s_{l+1} \leq$ bl and $l<n$

$04 \quad l=l+1$ 05 return $l$ 'is the maximal value' // line 05: return of the maximal $l$
// line 01: sorting of $\sigma$ // line 02: initialization of $l$ // line 03-04: computation of $l$

Theorem 39 Let $\mathrm{b}, \mathrm{l}$ and $\mathrm{n}$ be positive integers. Algorithm A-b-l-MAX computes the maximal $l$ for which $\sigma=\left(\mathrm{s}_{1}, \ldots, \mathrm{s}_{\mathrm{n}}\right)$ is $\mathrm{A}_{\mathrm{l}}^{\mathrm{b}}$-graphic in $\Theta(\mathrm{n})$ time.

Proof. Let $\mathrm{G}$ be a b-graph and $s^{\prime}=\left(s_{1}^{\prime}, \ldots, s_{n}^{\prime}\right)$ be the nonincreasingly sorted sequence consisting from the elements of $s . K_{l}^{b}$ contains $l$ vertices whose degrees are equal to $b(l-1)$. Therefore to find the maximal size $K_{l}^{b}$ which is a subgraph 
of $G$ it is sufficient to find the maximal $j$ satisfying $s_{j}^{\prime} \geq b(j-1)$. In lines $01-04$ A-b-l-Max computes this maximal $l$.

Lines 01 of A-b-l-Max requires $\Theta(n)$ time, lines 02 and $05 \mathrm{O}(1)$ time, and lines 03-04 require $\mathrm{O}(\mathrm{n})$ time, so the best and worst running times of this algorithm are both $\Theta(n)$.

As an example consider the sequence $\sigma=\left(\begin{array}{llll}6 & 6 & 1 & 6\end{array}\right)$ and $b=2$. Then $\sigma^{\prime}=\left(\begin{array}{llll}6 & 6 & 6 & 1\end{array}\right)$ and Ab-l-MAx returns with $l=3$. Indeed $G$ contains $K_{3}$ as a subgraph but it does not contain $\mathrm{K}_{4}$ as a subgraph. Since the sum of the elements of $s$ is odd, according to theorem of Erdős and Gallai [17] $s$ is not graphic, that is an $A_{l}^{b}$-graphic sequences are not always graphic.

\subsection{Algorithm J-b-l-MaX}

For given sequence $\sigma=\left(s_{1}, \ldots, s_{n}\right)$ of nonnegative integers and given nonnegative integer $b$ algorithm S-b-l-MAx computes the maximal $l$ for which the sequence $\sigma$ is $S_{l, n-l}^{b}$-graphic.

If $\mathrm{K}_{\mathrm{l}}^{\mathrm{b}}$ and $\mathrm{K}_{\mathrm{m}}^{\mathrm{b}}$ are vertex disjoint and $\mathrm{G}$ is the join of $\mathrm{K}_{\mathrm{l}}^{\mathrm{b}}$ and $\overline{\mathrm{K}}_{\mathrm{m}}$, then in $\mathrm{G}$ the degrees of the vertices of $K_{l}$ are equal to $b(l-1+m)$, while the degrees of the vertices of $\mathrm{K}_{\mathrm{m}}$ are equal to $\mathrm{bm}$. This observation is the base of the following algorithm S-b-l-MAX.

Input. $\mathrm{n}$ : the length of the degree sequence $\mathrm{s}$;

$\sigma=\left(s_{1}, \ldots, s_{n}\right):$ a sequence of nonnegative integers;

$\mathrm{b}$ : the maximal permitted number of edges between two different vertices.

Output. $l$ : the maximal value for which $\sigma$ is $S_{l, n-l}^{b}$-graphic or the message ' $\sigma$ is not $(b, l, n-l)$-graphic'.

Work variable. i: cycle variable.

$\operatorname{J-b}-\mathrm{l}-\operatorname{MAx}(n, \sigma, b)$

01 if $s_{1} / b$ is not integer

02 return ' $\sigma$ is not $(b, l, n-l)$-graphic'

// line 01-02: constant time test

03 Counting-Sort $(n, \sigma)$

$04 \mathrm{l}=1$

05 while $s_{l+1}==s_{1}$ and $l<n$

$06 \quad l=l+1$

07 if $s_{l+1} \neq b l \quad / /$ line $07-08: \sigma$ is not $(b, l, n-l)$-graphic

08 's is not $(\mathrm{b}, \mathrm{l}, \mathrm{n}-\mathrm{l})$-graphic'

09 return $l$ 'is the maximal value' 
Theorem 40 Algorithm J-b-l-Max computes the maximal $\mathrm{l}$ for which $\sigma=$ $\left(\mathrm{s}_{1}, \ldots, \mathrm{s}_{\mathrm{n}}\right)$ is $\left.\mathrm{S}_{(}^{\mathrm{b}} \mathrm{l}, \mathrm{n}-\mathrm{l}\right)$-graphic in $\Theta(\mathrm{n})$ time.

Proof. Let $b, l, m$ and $n$ be positive integers. Let $G$ be a $b$-graph and $\sigma=\left(s_{1}^{\prime}, \ldots, s_{n}^{\prime}\right)$ be the nonincreasingly sorted sequence consisting from the elements of $s^{\prime}$.

The next part of the proof is similar to the corresponding part of Theorem 39.

Line 01 of J-b-l-Max requires $\Theta(n)$ time and lines $02-05$ require $O(n)$ time, so the best running time is $\Theta(1)$ and the worst running time is $\Theta(n)$.

We remark that if the input of J-b-l-Max is sorted, then we can omit lines 03 and 04 , and using logarithmic search we can reduce the worst case running time to $\Theta(\log n)$.

\section{Acknowledgement}

The authors thank the useful remarks of the unknown referee and papers of Professor Jian-Hua Yin (Hainan University).

\section{References}

[1] S. M. de Almeida, C. P. de Mello, A. Morgana, Classification problem for split graphs, J. Brazilian Comp. Soc., 18, (2) (2012) 95-101. $\Rightarrow 266$

[2] M. D. Barrus, Hereditary unigraphs and Erdős-Gallai equalities, Discrete Math., 313, (21) (2013) 2469-2481. $\Rightarrow 263$

[3] D. Bauer, S. L. Hakimi, E. Schmeichel, Recognising of tough graphs is NP-hard, Discrete Appl. Math., 28 (1995) 191-195. $\Rightarrow 266$

[4] C. Benzaken, P. L. Hammer, D. De Werra, Split graphs of Dilworth number 2, Discrete Math., 55, (2) (1985) 123-127. $\Rightarrow 256$

[5] Z. Blázsik, M. Hujter, A. Pluhár, Zs. Tuza, Graphs with no induced $\mathrm{C}_{4}$ and $2 \mathrm{~K}_{2}$, Discrete Math., 115 (1993) 51-55. $\Rightarrow 256,263$

[6] E. Boros, V. A. Gurvich, I. Zverovich, On split and almost CIS-graphs, Australas. J. Combin., 43 (2009) 163-180. $\Rightarrow 255$

[7] A. Brandstädt, Partitions of graphs into one or two stable sets and cliques, Discrete Math., 152 (1996) 47-54. $\Rightarrow 254,256$

[8] A. Brandstädt, Corrigendum, Discrete Math., 186 (1998) 295-295. $\Rightarrow 254,256$

[9] A. Brandstädt, P. L. Hammer, V. B. Le, V. V. Lozin, Bisplit graphs, Discrete Mathematics 299 (2005) 11-32. $\Rightarrow 255,256$

[10] A. Brandstädt, V. B. Le, J. P. Spinrad, Graph Classes: A Survey, SIAM Monographs on Discrete Mathematics and Applications, SIAM, Philadelphia, PL, 1999. $\Rightarrow 254,256,266$ 
[11] R. E. Burkard, P. L. Hammer, A note on Hamiltonian split graphs, J. Comb. Graph Theory, Series B, 28 (1980) 245-248. $\Rightarrow 265$

[12] G. Chartrand, L. Lesniak, P. Zhang, Graphs and Digraphs, CRC Press, Boca Raton, FL, 2011. $\Rightarrow 253$

[13] V. Chungphaisan, Conditions for a sequences to be r-graphic, Discrete Math., 7 (1974) $31-39 . \Rightarrow 257,258$

[14] V. Chvátal, The toughness of graphs, Discrete Math., 5 (1973) 215-228. $\Rightarrow 266$

[15] R. J. Clarke, Covering a set by subsets, Discrete Math., 181 (1990) 147-152. $\Rightarrow$ 264

[16] T. H. Cormen, Ch. E. Leiserson, R. L. Rivest, C. Stein, Introduction to Algorithms Third edition, The MIT Press/McGraw Hill, Cambridge/New York, 2009. $\Rightarrow 275$

[17] P. Erdős, T. Gallai, Gráfok elöírt fokú pontokkal (Graphs with given degrees of vertices), Mat. Lapok, 11 (1960), 264-274. $\Rightarrow 253,256,258,280$

[18] P. Erdős, A. Gyárfás, Split and balanced colorings of complete graphs, Discrete Mathematics, 200, (1-3) (1999), 79-86. $\Rightarrow 264$

[19] P. Erdős, M. S. Jacobson, J. Lehel, Graphs realizing the same degree sequences and their respective clique numbers, in: Y. Alavi (Ed.), Graph Theory, Combinatorics and Applications, vol. 1, John Wiley and Sons, New York, 1991, 439-449. $\Rightarrow 264$

[20] T. Feder, P. Hell, S. Klein, R. Motwani, List partitions, SIAM J. Discrete Math, 16 (2003) 449-478. $\Rightarrow 278$

[21] S. Földes, P. Hammer, Split graphs having Dilworth number two, Canad. J. Math. 29, (3) (1977) 666-672. $\Rightarrow 254,256$

[22] S. Földes, P. Hammer, Split graphs, in (ed. E. Hoffman et al.) Proc. 8th SouthEastern Conf. Combinatorics, Graph Theory Comp. Congressus Num., XIX (1977) 311-315. $\Rightarrow 254,256,263$

[23] S. Földes, P. Hammer, The Dilworth number of a graph, Annals Discrete Math., 2 (1978) 211-219. $\Rightarrow 256$

[24] D. R. Fulkerson, A. J. Hoffman, M. H. McAndrew, Some properties of graphs with multiple edges, Canad. J. Math., 17 (1965) 166-177. $\Rightarrow 253,269,271$

[25] V. Gasharov, The Erdős-Gallai criterion and symmetric functions, Europ. J. Combinatorics, 18 (1997) 287-294. $\Rightarrow 258$

[26] M. C. Golumbic, Algorithmic Graph Theory and Perfect Graphs, Academic Press, 1980. $\Rightarrow 254,256,263,278$

[27] R. J. Gould, M. S. Jacobson, J. Lehel, Potentially G-graphical degree sequences, in: Y. Alavi (Ed.), Combinatorics, Graph Theory and Algorithms, vol. 1, New Issues Press, Kalamazoo, Michigan, 1999, 451-460. $\Rightarrow 261,264$

[28] J. L. Gross, J. Yellen, P. Zhang. Handbook of Graph Theory, CRC Press, Boca Raton, FL, 2013. $\Rightarrow 252,253,254,256$

[29] A. Gyárfás, J. Lehel, On-line and first fit colorings of graphs. J. Graph Theory, 12, (2) (1988) 217-227. $\Rightarrow 264$

[30] A. Gyárfás, Generalized split graphs and Ramsey numbers, J. Comb. Theory, Series A 81, (2) (1998) 255-261. $\Rightarrow 255,256$ 
[31] M. Habib, A. Mamcarz, Colored modular and split decompositions of graphs with applications to trigraphs, in (ed. D. Kratsch and I. Todinca) Graph-Theoretic Concepts in Computer Science (40th International Workshop, WG 2014, Nouanle-Fuzelier, France, June 25-27, 2014). Series: Lecture Notes in Computer Science, 8747, Springer Verlag, Berlin, 2014, 263-274. $\Rightarrow 266$

[32] S. L. Hakimi, On the realizability of a set of integers as degrees of the vertices of a simple graph. J. SIAM Appl. Math., 10 (1962), 496-506. $\Rightarrow 257,258$

[33] S. L. Hakimi, E. F. Schmeichel, Graphs and their degree sequences: A survey, in: Theory and Applications of Graphs, Lecture Notes in Math. 642, SpringerVerlag, Berlin 1978, 225-235. $\Rightarrow 261$

[34] P. L. Hammer, B. Simeone, The splittance of a graph, Combinatorica, 1, (3) (1981) 275-284. $\Rightarrow 263,264,278$

[35] P. Hanion, Enumeration of graphs by degree sequence, J. Graph Theory, 3 (1979) $295-299 . \Rightarrow 260$

[36] V. Havel, A remark on the existence of finite graphs (Czech), C̆asopis Pěst. Mat., 80 (1955), 477-480. $\Rightarrow 257,258$

[37] P. Heggerness, D. Kratsch, Linear-time certifying algorithms for recognizing split graphs and related graph classes, Nordic J. Comp., 14 (2007) 87-108. $\Rightarrow 278$

[38] P. Heggernes, F. Mancini, Minimal split completions. Discrete Appl. Math., 157, (12) (2009) 2659-2669. $\Rightarrow 266$

[39] A. P. Heinz, Total number of split graphs (chordal + chordal complement) on $\mathrm{n}$ vertices. In: (ed. N. J. A. Sloane): The On-Line Encyclopedia of the Integer Sequences. 2014. $\Rightarrow 265$

[40] P. Hell, D. Kirkpatrick, Linear-time certifying algorithms for near-graphical sequences, Discrete Math., 309, (18) (2009) 5703-5713. $\Rightarrow 260$

[41] P. Hell, S. Klein, F. Protti, L. Tito, On generalized split graphs, Electronic Notes Disc. Math., 7 (2001) 98-101. $\Rightarrow 255$

[42] L. Ibarra, Fully dynamic algorithms for chordal graphs and split graphs, ACM Trans. Algorithms, 4(4), (2008), Art. 40, 20 pages. $\Rightarrow 266$

[43] A. Iványi, Reconstruction of complete interval tournaments, Acta Univ. Sapientiae, Inform., 1, (1) (2009) 71-88. $\Rightarrow 252,253,256$

[44] A. Iványi, Reconstruction of complete interval tournaments II, Acta Univ. Sapientiae, Math., 2, (1) (2010) 47-71. $\Rightarrow 252,253$

[45] A. Iványi, Degree sequences of multigraphs, Annales Univ. Sci. Budapest., Rolando Eötvös Nom., Sectio Comp., 37 (2012) 195-214. $\Rightarrow 256,259,260$, 262

[46] A. Iványi, Z. Kása, Parallel enumeration of graphical sequences (in Hungarian), Alk. Mat. Lapok, 31, (2014) 1-58. $\Rightarrow 260$

[47] A. Iványi, L. Lucz, Degree sequences of multigraphs (in Hungarian), Alk. Mat. Lapok, 29, (2012) 1-54. $\Rightarrow 260$

[48] A. Iványi, L. Lucz, T. F. Móri, P. Sótér, On the Erdős-Gallai and Havel-Hakimi algorithms. Acta Univ. Sapientiae, Inform. 3, 2 (2011) 230-268. $\Rightarrow 256,259$, $260,262,275$ 
[49] A. Iványi, S. Pirzada. Comparison based ranking, in: ed. A. Iványi, Algorithms of Informatics, Vol. 3, mondAt, Vác, 2013, 1209-1258. $\Rightarrow 260$

[50] A. E. Kézdy, J. Lehel, Degree sequences of graphs with prescribed clique size. In: Y. Alavi et. al. (eds.) Combinatorics, Graph Theory, and Algorithms, vol. 2., Michigan, New Issues Press, Kalamazoo, 1999, 535-544. $\Rightarrow 260$

[51] D. J. Kleitman, D. L. Wang, Algorithm for constructing graphs and digraphs with given valences and factors, Discrete Math., 6 (1973) 79-88. $\Rightarrow 254,257$, 258

[52] D. Kratsch, R. M. McConnell, K. Mehlhorn, J. P. Spinrad, Certifying algorithms for recognizing interval graphs and permutation graphs, SIAM J. Comput., 36, (2) (2006) 326-353. $\Rightarrow 266$

[53] D. Kratsch, J. Lehel, H. Müller, Toughness, hamiltonicity and split graphs, Discrete Math., 150 (1996) 231-245. $\Rightarrow 266$

[54] M. D. Lamar, Split digraphs, Discrete Math., 312, (7) (2012) 1314-1325. $\Rightarrow 266$

[55] V. B. Le, H. N. de Ridder, Probe split graphs, Discrete Math. Theor. Comput. Sci., 9(1), (2007) 207-238. $\Rightarrow 255$

[56] J. S. Li, Z. X. Song, An extremal problem on the potentially $p_{k}$-graphic sequence, Discrete Math., 212 (2000) 223-231. $\Rightarrow 264$

[57] J. S. Li, Z. X. Song, R. Luo, The Erdős-Jacobson-Lehel conjecture on potentially $p_{k}$-graphic sequences is true, Sci. China Ser. A, 41 (1998) 510-520. $\Rightarrow 264$

[58] F. Maffray, M. Preissmann, Linear recognition of pseudo-split graphs, Discrete Appl. Math. 52 (1994) 307-312. $\Rightarrow 263,279$

[59] R. M. McConnell, K. Mehlhorn, S. Näher, P. Schweitzer, Certifying algorithms, Computer Science Review, 5, (2) (2011) 119-161. $\Rightarrow 260$

[60] C. McCreesh, Multi-threaded maximum clique, level 4 Project, School of Computing Science, of Glasgow University, 2013, 38 pages. $\Rightarrow 264$

[61] F. R. McMorris, C. Wang, P. Zhang, On probe interval graphs, Discrete Appl. Math., 88(1-3), (1998) 315-324. $\Rightarrow 255$

[62] S. D. Nikolopoulos, Constant-time parallel recognition of split graphs, Inf. Proc. Letters, 54, (1) (1995) 1-8. $\Rightarrow 265$

[63] P. M. Pardalos, J. Rappe, M. G. S. Resende, An exact parallel algorithm for the maximum clique problem, High Performance Algorithms and Software in Nonlinear Optimization, Applied Optimization, 24 (1998) 279-300. $\Rightarrow 264$

[64] P. M. Pardalos, J. Xue, The maximum clique problem, J. Global Opt. (1994) $301-328 . \Rightarrow 264$

[65] J. Peemüller, necessary conditions for hamiltonian split graphs, Discrete Math., 54 (1985) 45-57. $\Rightarrow 265,266$

[66] S. Pirzada, An Introduction to Graph Theory, Universities Press, Orient Blackswan, India, 2012. $\Rightarrow 253$

[67] S. Pirzada, B. A. Chat, Potentially graphic sequences of split graphs, Kragujevac J. Math., 38, (1) (2014) 73-81. $\Rightarrow 255,256,262,263$

[68] A. R. Rao, The clique number of a graph with given degree sequence, in: A. R. Rao (Ed.) Proc. Symp. on Graph Theory, MacMillan and Co. Limited, India, ISI Lecture Notes Series, 4 (1979) 251-267. $\Rightarrow 260$ 
[69] A. R. Rao, A survey of the theory of potentially P-graphic and forcibly P-graphic degree sequences, Comb. Graph Theory, Proc. Symp. (Calcutta 1980), Lect. Notes Math. 885 (1981) 417-440. $\Rightarrow 260$

[70] A. R. Rao, An Erdős-Gallai type result on the clique number of a realization of a degree sequence (unpublished). $\Rightarrow 260$

[71] G. F. Royle, Counting set covers and split graphs, J. Integer Sequences, 3 (2000), Article 00.2.6, 5 pages. $\Rightarrow 264$

[72] M. C. Schmidt, N. F. Samatova, K. Thomas, B.-H. Park, A scalable, parallel algorithm for maximal clique enumeration, J. Parallel Dist. Comp., 69, (4) (2009) 417-428. $\Rightarrow 264$

[73] P. S. Segundo, D. Rodríguez-Losada, A. Jiménez, An exact bit-parallel algorithm for the maximum clique problem, Computers \& Operations Res., 38, (2) (2011) $571-581 . \Rightarrow 264$

[74] N. J. A. Sloane, The number of degree-vectors for simple graphs. In (ed. N. J. A. Sloane): The On-Line Encyclopedia of the Integer Sequences. 2014, http://oeis.org/A004251 . $\Rightarrow 260$

[75] S. Szabó, Parallel algorithms for finding cliques in a graph, J. Physics: Conf. Series, 268, (1) (2011) 012030. $\Rightarrow 264$

[76] E. Tomita, A. Tanaka, H. Takahashi, The worst-case time complexity for generating all maximal cliques and computational experiments, Theoretical Computer Science, 363, (1) (2006) 28-42. $\Rightarrow 264$

[77] A. Tripathi, H. Tyagi, A simple criterion on degree sequences of graphs. Discrete Appl. Math., 156, 18 (2008) 3513-3517. $\Rightarrow 258$

[78] A. Tripathi, S. Venugopalan, D. B. West, A short constructive proof of the Erdős-Gallai characterization of graphic lists, Discrete Math., 310, (4) (2010) $843-844 . \Rightarrow 258$

[79] R. I. Tyshkevich, The canonical decomposition of a graph, Doklady Akademii Nauk SSSR (in Russian), 24 (1980) 677-679. $\Rightarrow 263$

[80] R. I. Tyshkevich, A. A. Chernyak, Yet another method of enumerating unmarked combinatorial objects, Mathematical Notes, 48, (6) (1990) 1239-1245 and (in Russian) Mat. Zametki, 48, (6) (1990) 98-105. $\Rightarrow 264$

[81] R. I. Tyshkevich, O. I. Melnikow, V. M. Kotov, On graphs and degree sequences: the canonical decomposition (in Russian), Kibernetica, 6 (1981) 5-8. $\Rightarrow 263$

[82] Wikipedia, Split graph. http://en.wikipedia.org/wiki/Split_graph, 2014. $\Rightarrow 256$, 263

[83] G. J. Woeginger, The taughness of split graphs, Discrete Math., 190 (1998) 195-197. $\Rightarrow 266$

[84] J.-H. Yin, Conditions for $r$-graphic sequences to be potentially $K_{m+1}^{(r)}$-graphic, Discrete Math., 309 (2009) 6271-6276. $\Rightarrow 261$

[85] J.-H. Yin, A generalization of a conjecture due to Erdős, Jacobson and Lehel, Discrete Math., 309 (2009) 2579-2583. $\Rightarrow 261,264$

[86] J.-H. Yin, A Rao-type characterization for a sequence to have a realization containing a split graph, Discrete Math., 311 (2011) 2485-2489. $\Rightarrow 255,256$ 
[87] J.-H. Yin, A Havel-Hakimi type procedure and a sufficient condition for a sequence to be potentially $\mathrm{S}_{\mathrm{r}, \mathrm{s}}$-graphic, Czechoslovak Math. J., 62, (3) (2012) 863-867. $\Rightarrow 255,256,262,279$

[88] J.-H. Yin, An extension of A. R. Rao's characterization of potentially $\mathrm{K}_{\mathrm{m}+1^{-}}$ graphic sequences, Discrete Appl. Math., 161, (7-8) (2013) 1118-1127. $\Rightarrow 256$, 262

[89] J.-H. Yin, A Rao-type characterization for a sequence to have a realization containing an arbitrary subgraph H, Acta Math. Sin., 30, (3) (2014) 389-394. $\Rightarrow$ 262

[90] J.-H. Yin, Y.-S. Li, Two sufficient conditions for a graphic sequence to have a realization with prescribed clique size, Discrete Math., 209 (2005) 218-227. $\Rightarrow$ 264

[91] B. Zavalnij, Three versions of clique search parallelization, J. Comp. Sci. Inf. Technology, 2(2), (2014) 9-20. $\Rightarrow 264$

Received: July 20, 2014 • Revised: November 16, 2014 